\title{
CD47 blockade augmentation of trastuzumab antitumor efficacy dependent on antibody-dependent cellular phagocytosis
}

\author{
Li-Chung Tsao, ${ }^{1}$ Erika J. Crosby, ${ }^{1}$ Timothy N. Trotter, ${ }^{1}$ Pankaj Agarwal, ${ }^{1}$ Bin-Jin Hwang, ${ }^{1}$ \\ Chaitanya Acharya, ${ }^{1}$ Casey W. Shuptrine, ${ }^{1}$ Tao Wang, ${ }^{1}$ Junping Wei, ${ }^{1}$ Xiao Yang, ${ }^{1}$ Gangjun Lei, ${ }^{1}$ \\ Cong-Xiao Liu, ${ }^{1}$ Christopher A. Rabiola, ${ }^{1}$ Lewis A. Chodosh, ${ }^{2}$ William J. Muller, ${ }^{3}$ \\ Herbert Kim Lyerly, ${ }^{1,4,5}$ and Zachary C. Hartman ${ }^{1,5}$ \\ 'Department of Surgery, Duke University, Durham, North Carolina, USA. ${ }^{2}$ Department of Cancer Biology, University of \\ Pennsylvania, Philadelphia, Pennsylvania, USA. ${ }^{3}$ Department of Biochemistry, McGill University, Montreal, Quebec, \\ Canada. ${ }^{4}$ Department of Immunology, and ${ }^{5}$ Department of Pathology, Duke University, Durham, North Carolina, USA.
}

The HER2-specific monoclonal antibody (mAb), trastuzumab, has been the mainstay of therapy for HER $2^{+}$breast cancer (BC) for approximately 20 years. However, its therapeutic mechanism of action (MOA) remains unclear, with antitumor responses to trastuzumab remaining heterogeneous and metastatic HER2+ BC remaining incurable. Consequently, understanding its MOA could enable rational strategies to enhance its efficacy. Using both murine and human versions of trastuzumab, we found its antitumor activity dependent on Fc $\gamma$ receptor stimulation of tumorassociated macrophages (TAMs) and antibody-dependent cellular phagocytosis (ADCP), but not cellular cytotoxicity (ADCC). Trastuzumab also stimulated TAM activation and expansion, but did not require adaptive immunity, natural killer cells, and/or neutrophils. Moreover, inhibition of the innate immune ADCP checkpoint, CD47, significantly enhanced trastuzumab-mediated ADCP and TAM expansion and activation, resulting in the emergence of a unique hyperphagocytic macrophage population, improved antitumor responses, and prolonged survival. In addition, we found that tumor-associated CD47 expression was inversely associated with survival in HER2 ${ }^{+}$ BC patients and that human HER2+ BC xenografts treated with trastuzumab plus CD47 inhibition underwent complete tumor regression. Collectively, our study identifies trastuzumab-mediated ADCP as an important antitumor MOA that may be clinically enabled by CD47 blockade to augment therapeutic efficacy.

Conflict of interest: An invention disclosure at Duke University's Office of Licensing and Ventures has been generated for "identifying phagocytic macrophage as a biomarker" by the following authors: $\mathrm{LT}, \mathrm{ZH}$, and $\mathrm{HKL}$ (patent T-006575).

Copyright: (c) 2019, American Society for Clinical Investigation.

Submitted: July 21, 2019

Accepted: October 31, 2019

Published: December 19, 2019

Reference information: JCI Insight 2019;4(24):e131882.

https://doi.org/10.1172/jci.

insight.131882.

\section{Introduction}

Approximately $20 \%$ of breast cancer (BC) overexpresses HER2, recognized as an oncogenic driver of an aggressive cancer phenotype with a poor prognosis $(1,2)$. Monoclonal antibodies (mAbs) targeting HER2 were developed in the 1980s to inhibit HER2 oncogenic signaling, leading to the clinical development and regulatory approval of trastuzumab in 1998 for metastatic HER2-overexpressing BC, followed by clinical trials of trastuzumab use in the adjuvant setting. Following its approval, additional HER2-targeting mAbs have also been generated to improve outcomes $(3,4)$. However, the clinical benefit associated with HER2 $\mathrm{mAb}$ therapies in patients with HER2-overexpressing $\mathrm{BC}$ remains heterogeneous and metastatic HER2 ${ }^{+} \mathrm{BC}$ remains incurable $(5,6)$. Consequently, mechanistic studies of the antitumor mechanism(s) of action (MOA) of trastuzumab and its resistance remain critical, not only to improve outcomes in patients with HER2 ${ }^{+} \mathrm{BC}$ but also to gain insight into mechanisms that would extend $\mathrm{mAb}$ therapies to other types of cancers.

While suppression of HER2 signaling was a primary focus of early mechanistic studies, subsequent studies also focused on the role of immunity in mediating the antitumor effects of trastuzumab (7). In particular, studies have shown that the interaction of anti-HER2 antibodies with Fc $\gamma$ receptors (FCGRs) expressed on innate immune cells such as macrophages, monocytes, natural killer (NK) cells, and dendritic cells may be involved in its therapeutic activity $(8,9)$. The consequences of crosstalk with FCGR-bearing 
immune cells (8-10) are supported by the clinical observation that some host FCGR polymorphisms are associated with improved clinical outcome in HER2 ${ }^{+}$BC patients treated with trastuzumab (11). Specifically, several studies have suggested the importance of these receptors in mediating antibody-dependent cellular cytotoxicity (ADCC), through NK cells or neutrophils for trastuzumab efficacy $(8,9,12-14)$. However, other studies have suggested the importance of adaptive immunity in mediating trastuzumab efficacy, indicating that $\mathrm{T}$ cells may be critical for its antitumor MOA $(8,15)$.

While multiple MOAs involving either innate or adaptive immunity are possible, an underexplored mechanism is through mAb engagement of FCGRs to stimulate macrophage-mediated antibody-dependent cellular phagocytosis (ADCP). Inconsistent reports about the role of ADCP exist, with a recent study demonstrating the ability of trastuzumab to elicit ADCP (16), while another study suggests that trastuzumab-mediated ADCP triggers macrophage immunosuppression in HER2 $2^{+} \mathrm{BC}(17)$. These disparate results may be partially attributed to the use of a wide range of tumor models (many not specifically driven by active HER2 signaling), as well as the use of different HER2-specific mAb clones of varied isotypes, which can elicit a range of different responses from various FCGRs $(18,19)$. Thus, the immunologic basis for the activity of trastuzumab remains inconclusive, but could be effectively investigated through the development and use of appropriate HER2-targeting mAbs and model systems.

In this study, we developed and utilized fully murinized trastuzumab mAbs (clone 4D5) with isotypes of different activating-to-inhibitory ratios (A/I ratios, calculated by dividing the affinity of a specific IgG isotype for an activating receptor by the affinity for the inhibitory receptor) (19), as well as clinical-grade trastuzumab, to determine the MOA for trastuzumab antitumor efficacy. These mAbs were tested in multiple settings to interrogate ADCC and ADCP, as well as the impact on HER2 signaling and complement-dependent cytotoxicity (CDC). To determine the antitumor efficacy of these HER2 mAbs, we employed orthotopic implantation of HER2 ${ }^{+}$murine BC cells (transformed using a constitutively active isoform of human HER2) in immunocompetent models, as well as $\mathrm{Fcgr}^{-{ }^{-}}$, immune-deficient backgrounds, and human HER2 ${ }^{+}$BC xenograft models. In addition, we utilized a transgenic HER2 ${ }^{+}$BC model driven by an oncogenic isoform of human HER2 to simulate an endogenous mammary tumor immune microenvironment $(20,21)$. Collectively, these studies revealed an essential role for tumor-associated macrophages (TAMs) in mediating the therapeutic activity of trastuzumab through promoting ADCP of HER2 ${ }^{+}$ tumor cells without evidence for significant induction of adaptive T cell responses against HER2. We also observed that this effect was subverted by innate mechanisms of immunosuppression in the tumor microenvironment that limit macrophage ADCP.

Previous studies have demonstrated that ADCP is principally regulated by antiphagocytic “don't eat me" signals that are amplified in many cancers $(22,23)$. Chief among these is CD47, which has been shown to be highly expressed in different cancers and functions to suppress phagocytosis through binding to and triggering signaling of macrophage $\operatorname{SIRP} \alpha(23,24)$. Notably, CD47 expression is also upregulated in $\mathrm{BC}$ (25). As a potential means to subvert innate immune regulation and enhance ADCP and possibly alter the macrophage phenotype in HER2 ${ }^{+} \mathrm{BC}$, we also targeted the CD47/SIRP $\alpha$ innate immune checkpoint. In this study, we demonstrate that TAM ADCP can be significantly enhanced by blocking the CD47/SIRP $\alpha$ checkpoint to enable trastuzumab-mediated macrophage phagocytosis of HER2 ${ }^{+}$tumor cells. Collectively, these findings support the importance of the ADCP MOA, as well as suggest the therapeutic potential of utilizing $\mathrm{CD} 47 / \mathrm{SIRP} \alpha$ checkpoint blockade in combination with trastuzumab in HER2 ${ }^{+} \mathrm{BC}$ and potentially in other resistant HER2 ${ }^{+}$cancers (i.e., gastric, bladder, etc.) (26).

\section{Results}

Generation of murine trastuzumab (4D5) and its antitumor dependence on ADCP by TAMs. Trastuzumab was based on a HER2-specific mouse IgG1 mAb (4D5-IgG1, low A/I ratio), which was subsequently humanized to a human IgG1 isotype (high A/I ratio) that allows for superior activation of Fc receptors (27). Thus, to accurately study the function of trastuzumab in an immunocompetent mouse model, we constructed a murine 4D5 $\mathrm{mAb}$, but using the IgG2A isotype (4D5-IgG2A, high A/I ratio; Figure 1A) to better approximate an Fc-receptor-activating murine version of trastuzumab $(18,19,28)$. Unsurprisingly, we found that 4D5-IgG2A HER 2 binding is equivalent to trastuzumab (Supplemental Figure 1A; supplemental material available online with this article; https://doi.org/10.1172/jci.insight.131882DS1). This allowed us to interrogate the importance of the HER2-antibody Fc region as well as minimize the humoral immune responses against trastuzum$\mathrm{ab}$, a human antibody, when administered into a murine host (Supplemental Figure 1B). 
To test the antitumor efficacy of 4D5-IgG2A, we began by interrogating its impact on oncogenic HER2 signaling. As HER2 is weakly transformative in most cell lines, we employed a highly oncogenic isoform of human HER2 (HER2 $\triangle 16$ ) that constitutively dimerizes to create a transformed BALB/c mammary cell line dependent on HER2 signaling (21). In studies using HER2 $\Delta 16$, we observed that both 4D5-IgG2A and trastuzumab could suppress HER2 signaling (although not as potently as lapatinib; Supplemental Figure $1, \mathrm{C}$ and D), but not significantly enough to prevent tumor cell growth in vitro (Supplemental Figure 1E). This is in line with several recent studies, suggesting that the impact of trastuzumab is mediated through immune-based mechanisms $(29,30)$. Using transformed MM3MG-HER2 $\Delta 16$ as a model for HER2-driven $\mathrm{BC}$ growth in vivo, we next implanted these cells in the mammary fat pad of immunocompetent BALB/c mice. Tumor-bearing mice were treated weekly with 4D5-IgG2A or clinical-grade trastuzumab to determine if they could suppress tumor growth in an immunocompetent context. We found that both 4D5-IgG2A and trastuzumab significantly suppressed HER2 ${ }^{+}$BC growth, demonstrating that murine IgG2A was capable of significant antitumor activity (Figure 1B). Notably, we observed that 4D5-IgG2A and trastuzumab significantly increased the levels of TAMs (Figure 1C), but did not increase other immune infiltrates such as NK cells and T cells (Supplemental Figure 2A). Furthermore, using IFN- $\gamma$ ELISPOT assays we found that 4D5-IgG2A and trastuzumab treatment had no effect on systemic adaptive $\mathrm{T}$ cell responses against human HER2 epitopes (Supplemental Figure 2, B and C). In agreement with published reports (12), we observed that NK cell-mediated ADCC was increased by 4D5 or trastuzumab treatment in coculture systems (Supplemental Figure 2D). To determine if NK cells and/or adaptive immune cells mediate antitumor immunity in vivo, we next tested the HER2 mAb's ability to suppress HER2 ${ }^{+}$BC growth in T cell-, B cell-, and NK cell-deficient SCID-beige mice. In contrast with published reports (8), we found surprisingly no change in its antitumor efficacy (Figure 1D), suggesting the roles of adaptive immune and NK cells are minimal in trastuzumab/4D5 action in our in vivo model system. As neutrophil levels $\left(\mathrm{LY}_{6 \mathrm{G}}{ }^{+} \mathrm{CD} 11 \mathrm{~b}^{+}\right)$ were suppressed (Supplemental Figure 2A) and previous studies have also implicated neutrophils in trastuzumab-mediated immunity (14), we next depleted neutrophils using anti-LY6G in SCID-beige studies (Supplemental Figure 3, D and E), but did not observe any difference in antitumor efficacy (Figure 1E). To investigate the possible role of CDC, we performed CDC assays in vitro and found that neither 4D5-IgG2A nor trastuzumab was able to induce CDC in comparison with polyclonal HER2 antibodies, in line with other studies of trastuzumab (31) (Supplemental Figure 2E).

The increase in TAM levels after treatment suggested a functional role in trastuzumab antitumor immunity. We therefore implemented several strategies to deplete macrophages in our SCID-beige HER2 ${ }^{+}$ BC model. Using a prolonged anti-CSF1R antibody injection strategy (32), we achieved significant reduction of TAMs, which also limited TAM increases in 4D5-treated tumors (Figure 1F). Importantly, the reduction in TAM levels resulted in a significant decrease in HER2 mAb therapeutic efficacy (Figure 1G). We also utilized clodronate liposome injection to deplete macrophages in this model, but found we could only readily deplete macrophages in systemic circulation and not those in the tumor (Supplemental Figure 3A). Interestingly, this depletion had no effect on HER2 mAb therapy (Supplemental Figure 3, B and C), suggesting that macrophages in the mammary tumor are the major antitumor effectors. To explore the efficacy of macrophage-mediated HER2-specific antitumor activity, we established a bone marrow-derived macrophage (BMDM) coculture system to investigate the relative ADCC and ADCP activity mediated by 4D5-IgG2A and trastuzumab (33). Using latrunculin A, an inhibitor of actin polymerization that therefore blocks phagocytosis of immune complexes (34), we revealed that the dominant antitumor activity of HER2 mAbs mediated by macrophages is through ADCP (Figure 1H). Concanamycin A, an V-ATPase inhibitor reported to also inhibit perforin and cytotoxicity (35), had no effect on HER2 mAb activities. Collectively, these results suggested that trastuzumab therapy modifies the tumor microenvironment by promoting TAM expansion, and that the dominant mechanism of action by trastuzumab is mediated by ADCP of HER2 ${ }^{+}$tumor cells by macrophages.

The ADCP activity of $4 D 5$ requires the engagement with FCGRs and is isotype dependent. To further validate the mechanism of ADCP by 4D5-IgG2A treatment, we utilized Fcerlg-/- animals to test the requirement for FCGR engagement on phagocytic immune cells. Using macrophages cultured from $F_{c e r} 1 g^{-/}$and control mice, in vitro ADCP assays revealed that FCGRs on macrophages are critical for 4D5-induced ADCP of HER2 $^{+}$BC (Figure 2A). Accordingly, we found that the in vivo antitumor efficacy of 4D5-IgG2A therapy is mostly ablated in Fcer $1 g^{-1-}$ mice (Figure 2B). Importantly, FCGR expression was also required for macrophage expansion by 4D5-IgG2A in the tumor microenvironment (Figure 2C). 
A

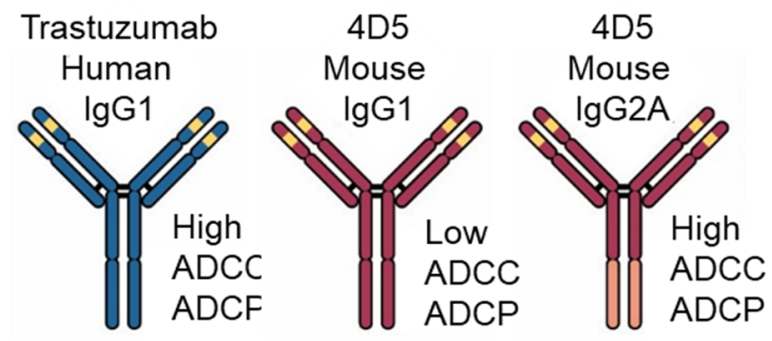

C
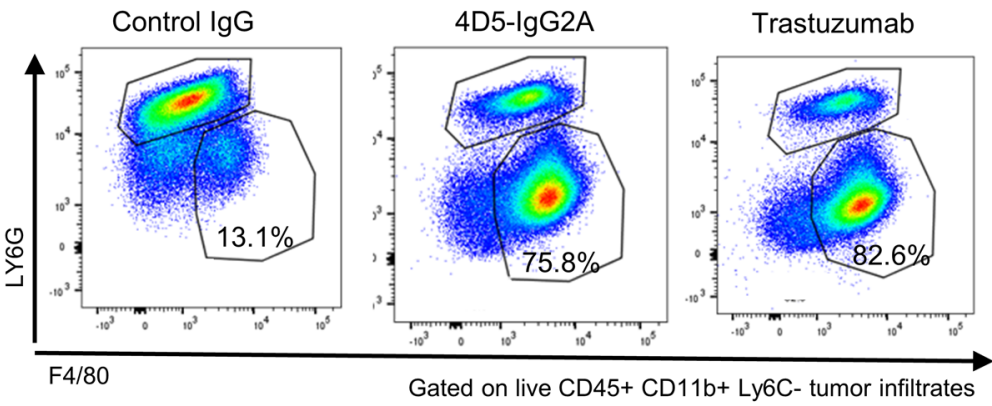

B MM3MG-HER2 16 growth in Balb/c mice

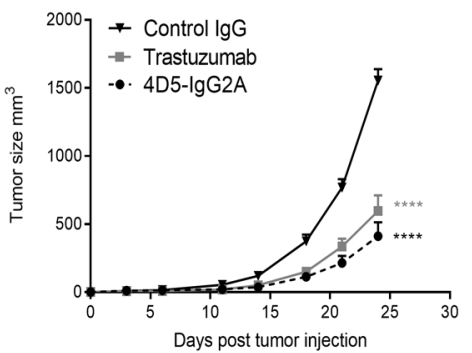

Tumor-associated macrophages (TAMs)

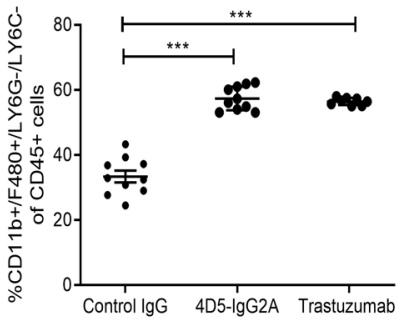

D

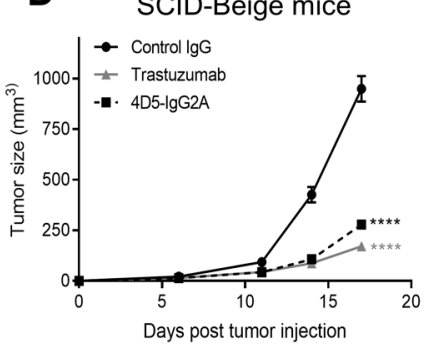

E

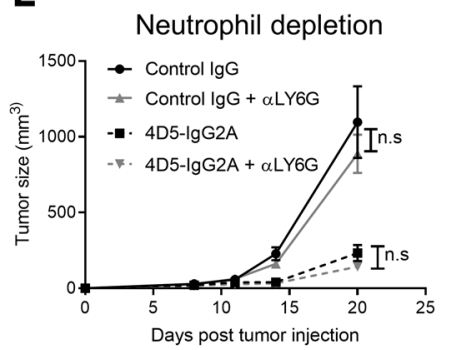

$\mathbf{F}$

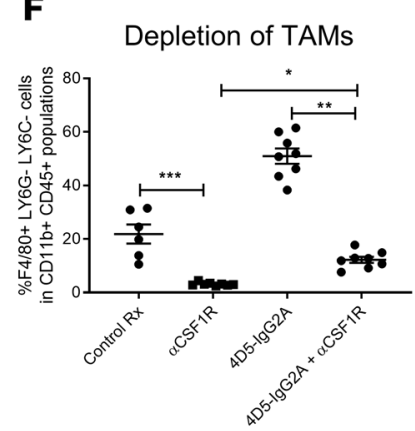

G

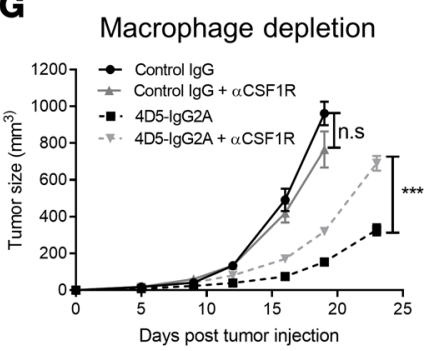

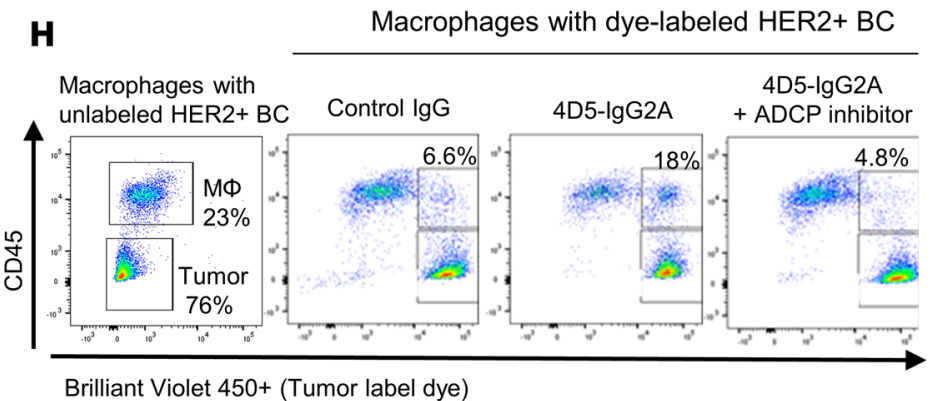
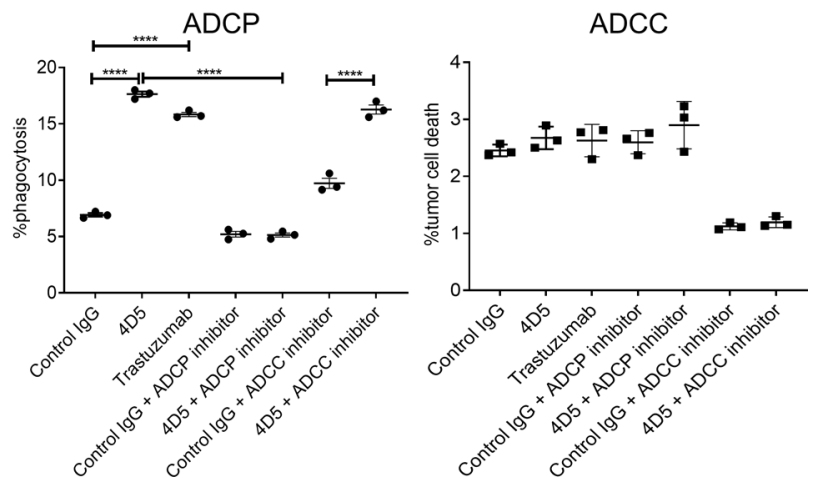

Figure 1. Generation of murine trastuzumab and its antitumor dependence on antibody-dependent cellular phagocytosis (ADCP) by tumor-associated macrophages (TAMs). (A) Cartoon representation of trastuzumab and 4D5 antibodies used in this study. (B) MM3MG cells expressing human HER2 16 were implanted into the mammary fat pads $\left(1 \times 10^{6}\right.$ cells) of BALB/c mice. Trastuzumab (human lgG1) or 4D5 (mouse lgG2A) was administered weekly (200 $\mu \mathrm{g}$

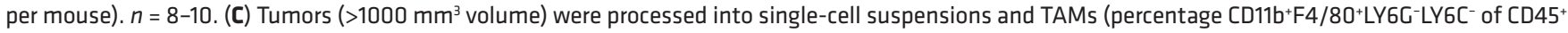
cells) were analyzed by FACS. $n=8-10$. (D) Experiment as in B was repeated in SCID-beige animals. $n=8$. (E) Experiment in SCID-beige was repeated using neutrophil-depleting anti-LY6G antibodies (clone IA8, $300 \mu \mathrm{g}$ per mouse biweekly). (F and $\mathbf{G}$ ) To deplete macrophages, SCID-beige mice were pretreated with anti-CSF1R antibody (clone AFS98, $300 \mu \mathrm{g}$, 3 times per week) for 2 weeks. (F) Macrophage depletion was verified by FACS. (G) 4D5-IgG2A was injected with anti-CSF1R treatment maintained throughout the experiment. $n=8$. (H) Trastuzumab/4D5 induced ADCP of HER2 ${ }^{+}$breast cancer (BC) cells by bone marrowderived macrophages (BMDMs). MM3MG-HER2 16 cells were labeled with Brilliant Violet 450 Dye and cocultured with BMDMs (3:1 ratio) with control or anti-HER2 antibodies $(10 \mu \mathrm{g} / \mathrm{mL})$. ADCP rates were measured as percentage of BMDM uptake of labeled tumor cells (CD45 ${ }^{+}$and $\left.\mathrm{BV}^{2} 50^{+}\right)$, and antibody-dependent cellular cytotoxicity (ADCC) rates were measured as percentage of dying free tumor cells (CD45- and LIVE/DEAD+). ADCP inhibitor (latrunculin A) or ADCC inhibitor (concanamycin A) was added as assay control. $n=3$; experiment was repeated 3 separate times. In $\mathbf{B}, \mathbf{D}, \mathbf{E}$, and $\mathbf{G}$, tumor growth was determined with caliper-based tumor measurement over time. Significance was determined by 2-way ANOVA with Tukey's multiple-comparisons test (C, F, and H) or 1-way ANOVA test with Tukey's multiple-comparisons test. All data represent the mean \pm SEM. ${ }^{*} P<0.05 ;{ }^{* *} P<0.01 ;{ }^{* *} P<0.001 ;{ }^{* * * *} P<0.0001$. 
These data demonstrated that HER2 mAb engagement with macrophage FCGRs is required for ADCP activity. Among the 4 mouse FCGRs, FCGR4 is the predominant FCGR mediating macrophage ADCP, plays a central role for mouse IgG2A activity, and has also been shown to exhibit the strongest binding affinity for trastuzumab $(16,36-38)$. To determine the impact of HER2-mAb isotype on FCGR4 engagement and antitumor efficacy, we compared the efficacy of 4D5-IgG1 (low A/I ratio) and compared its antitumor efficacy with 4D5-IgG2A (Figure 1A). We found that unlike 4D5-IgG2A, which elicited significant antitumor effects in vivo and $\mathrm{ADCP}$ in vitro, $4 \mathrm{D} 5-\mathrm{IgG} 1$ had no effect against HER2 ${ }^{+} \mathrm{BC}$ in vivo (Figure $2 \mathrm{D}$ ) and was inferior in promoting tumor ADCP by BMDMs (Figure 2E). To determine HER2-mAb isotype impact on FCGR4 and other activating FCGRs directly, we developed a mouse NFAT-luciferase assay that reports FCGR signaling and activation based on published methods (39). In agreement with established literature on mouse IgG subclasses and FCGR biology $(18,19,40)$, we found that 4D5-IgG2A engages with all 3 activating FCGRs, whereas 4D5-IgG1 only weakly activates FCGR3 (Figure 2, F-H). Additionally, mouse FCGR1 and FCGR4 have strong human-murine cross-reactivity with clinical-grade human trastuzumab (human IgG1 isotype), as reported previously (40), thus potentially explaining its in vivo efficacy in mice. Collectively, these results illustrate that the HER2 mAb's antitumor activity requires the successful engagement and activation of FCGRs on macrophages to induce ADCP.

CD47 blockade increases therapeutic efficacy of 4D5 and augments TAM expansion and phagocytosis. Our findings strongly supported an ADCP MOA for trastuzumab antitumor efficacy, which suggests that strategies to enhance ADCP may be synergistic with trastuzumab therapies. As previous studies have demonstrated that blockade of $\mathrm{CD} 47 / \mathrm{SIRP} \alpha$ can enhance mAb therapeutic efficacy, we investigated if targeting this ADCP-specific axis would enhance HER2 mAb ADCP without affecting ADCC activity. To begin our investigation, we documented the elevated expression of $C d 47$ in our MM3MG-HER2 16 tumors and generated CD47-KO cells (Supplemental Figure 4A) to determine the contribution of this axis to ADCP and $\mathrm{ADCC}$ in vitro. We observed that CD47-KO tumor cells exhibited generally enhanced ADCP that was significantly enhanced by HER2 mAbs, but had no effect on ADCC (Figure 3A). Additionally, we found that 4D5-mediated ADCP of CD47-KO tumors elicited the expression of proinflammatory cytokines and chemokines by macrophages (e.g., IL-6, TNF- $\alpha$, CCL3, CCL4, etc.), presumably due to enhanced ADCP activity (Figure 3B and Supplemental Figure 5). This demonstrates that 4D5-IgG2A alone triggers ADCP but was insufficient to stimulate significant proinflammatory activation within macrophages. However, upon blockade of the CD47 negative-regulatory axis, ADCP and an associated proinflammatory phenotype was significantly enhanced in macrophages.

As CD47 directly altered ADCP and macrophage activation in vitro, we next evaluated the impact of CD47-KO expression on tumor growth and HER2 mAb therapy in vivo. We found that CD47-KO HER2 ${ }^{+} \mathrm{BC}$ cells showed a delayed growth when implanted into mice, and were substantially more susceptible to 4D5-IgG2A inhibition (Figure 3C). Furthermore, we found significantly elevated TAM levels in CD47-KO tumors compared with the control tumors after 4D5-IgG2A treatment (Figure 3D). In a reciprocal approach, we overexpressed Cd47 in the tumor cells (Supplemental Figure 4B) and found this increased tumor resistance to 4D5-IgG2A therapy (Figure 3E) and prevented TAM increases (Figure 3F). These 2 genetic approaches validated the role of CD47 in suppressing trastuzumab's ADCP-mediated antitumor activity, and suggest blockade of CD47 could unleash the full potential of trastuzumab therapeutic efficacy by altering macrophage activation and expansion.

As recent studies have suggested CD47 blockade antibodies can elicit clinical responses (41), we next wanted to determine if CD47 blockade could enhance trastuzumab efficacy. Thus, we combined 4D5-IgG2A mAb with CD47 blockade antibody MIAP410 in immunocompetent mice bearing the MM3MGHER2 $\triangle 16$ tumors. While 4D5-IgG2A and CD47 blockade monotherapies both showed therapeutic efficacy, their combination significantly suppressed tumor growth more effectively than either 4D5-IgG2A or CD47 alone and also further increased TAM levels (Figure 4, A and B, and Supplemental Figure 7). In contrast, we observed that levels of other infiltrating immune cell types, except for regulatory $\mathrm{T}$ cells, were not significantly increased by weekly treatment of 4D5-IgG2A with CD47 blockade (Supplemental Figure 6A). As regulatory $\mathrm{T}$ cells were altered, we speculated that adaptive immune responses could also play a role in these enhanced responses. To explore the impact of adaptive immunity in the context of CD47 blockade, we repeated our in vivo experiments in adaptive immune-deficient SCID-beige mice (Figure 4C). As before, we observed a strong combinatorial effect between HER $2 \mathrm{mAb}$ and CD47 blockade, suggesting adaptive immunity and NK cells were not essential to the enhanced response with this combination therapy. Also as before, we found that CD47 blockade with 4D5-IgG2A further increased TAMs levels (Figure 4D), suggesting that relieving the CD47 checkpoint specifically promotes macrophage expansion and phagocytosis in tumors. 
A

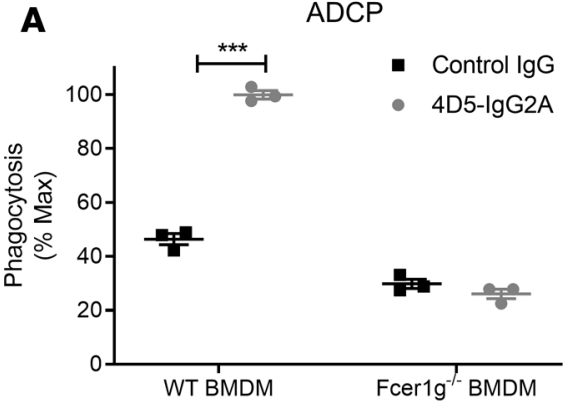

C
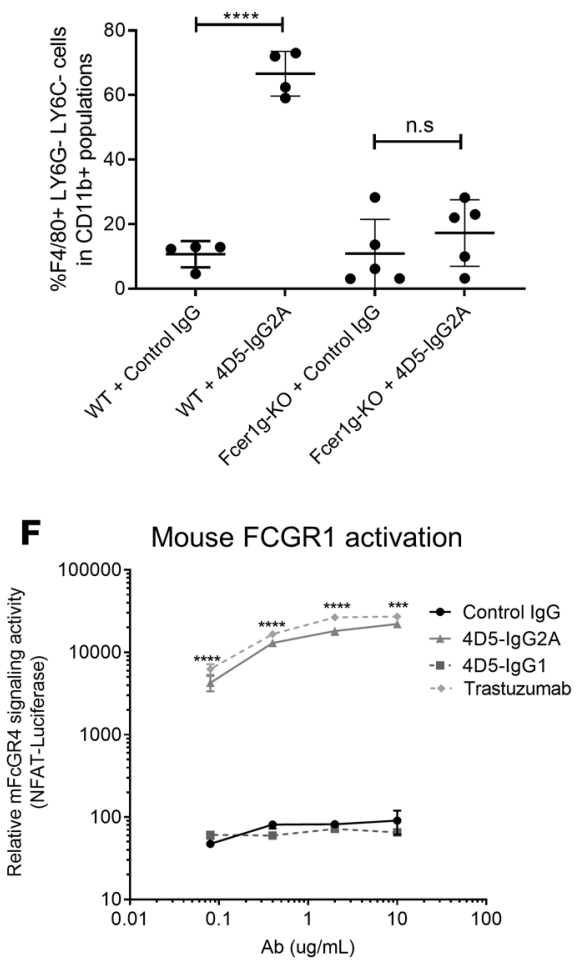

B

Wild type Balb/c

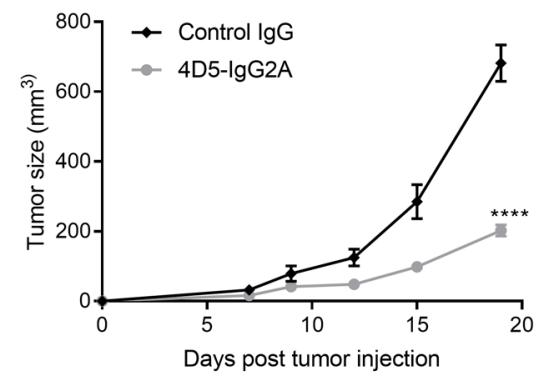

D

D MM3MG-HER2A16 growth

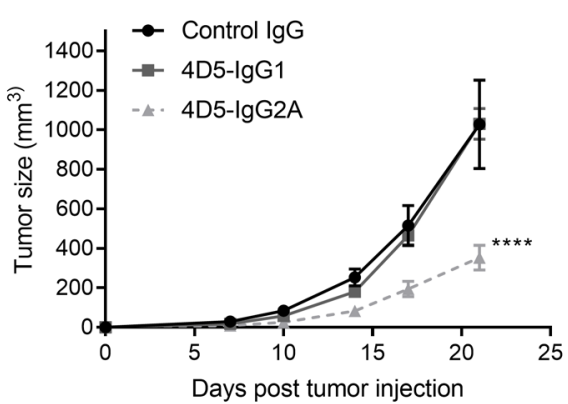

Fcer1g-/ Balb/c

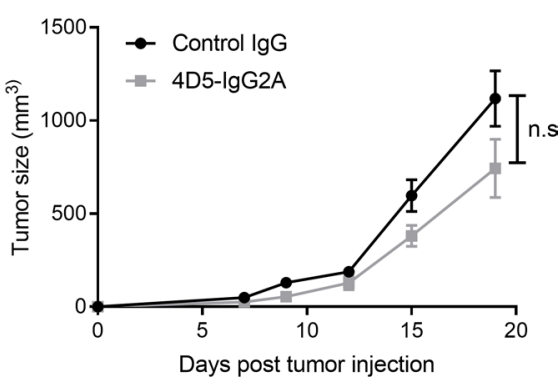

E

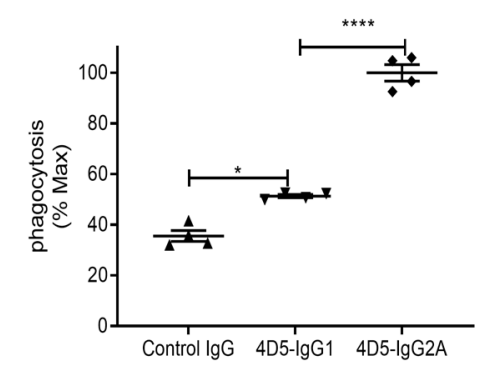

\section{G}

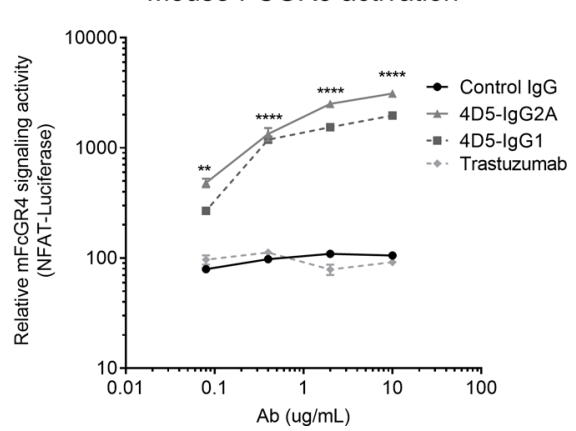

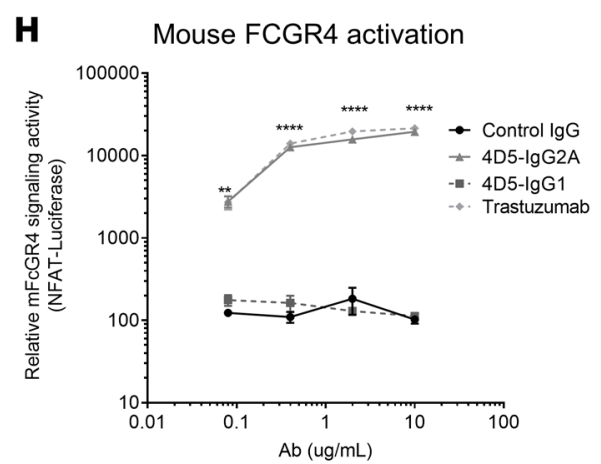

Figure 2. Antibody-dependent cellular phagocytosis (ADCP) of mouse trastuzumab (4D5) requires engagement with Fcy receptors (FCCRs) and is IgG2A isotype dependent. (A) FCCRs are required for 4D5-induced ADCP of HER2 $2^{+}$breast cancer cells by bone marrow-derived macrophages (BMDMs) in vitro. BMDMs were generated from wild-type and Fcer1 ${ }^{-1-}$ mice, and ADCP experiments were performed with the conditions described in Figure $1 \mathrm{E}$. $n=3$. (B and C) FCGR is required for the antitumor activity of $4 D 5$ therapy. (B) Wild-type or Fcer1g ${ }^{-1-}$ BALB/c mice were implanted with MM3MG-HER2 $\triangle 16$ cells as before (Figure 1B). 4D5-IgG2A or control antibodies were administered weekly (200 $\mu$ g per mouse intraperitoneally) and tumor growth was measured. $n=$ 5. (C) Tumor-associated macrophages (TAMs) from tumors in Figure 2B were analyzed by FACS. $n=4$ or 5. (D-F) The ADCP activity of 4D5 is IgG2A isotype dependent. (D) MM3MG-HER2 16 tumor growth in mice was repeated using 4D5 antibodies containing mouse lgG1 as a comparison to the previously utilized IgC2A isotype. $n=8-10$. (E) ADCP experiments with BMDM cultures were performed using 4D5-IgG1 versus 4D5-IgC2A antibody isotypes. $n=4$. (F-H) Mouse FCGR signaling activation assay. MM3MG breast cancer cells expressing HER2 were plated and treated with indicated antibody concentrations for 1 hour. Jurkat cells containing NFAT-luciferase reporter and expressing mouse FCGR1 (F), FCGR3 (C), or FCGR4 (H) were added to the target cells containing antibodies and cocultured for 4 hours. FCGR signaling activation was assessed by luciferase activity quantification. $n=4$. Significance was determined by 1-way ANOVA with Tukey's multiple-comparisons test (A, C, and E) or 2-way ANOVA with Tukey's multiple-comparisons test vs. control IgG group (B, D, and $\mathbf{F}-\mathbf{H})$. All data represent the mean \pm SEM. ${ }^{*} P<0.05 ;{ }^{* *} P<0.01 ;{ }^{* *} P<0.001 ;{ }^{* * *} P<0.0001$.

In order to directly demonstrate tumor $\mathrm{ADCP}$ by endogenous macrophages in the tumor microenvironment, we labeled MM3MG-HER2 $\Delta 16$ tumor cells with DiD dye (a carbocyanine membrane-binding probe) prior to implantation, a strategy to detect phagocytosis of labeled target cells in vivo (42). When the tumors reached a volume of approximately $1000 \mathrm{~mm}^{3}$, we treated the animals with 4D5-IgG2A antibody or in combination with CD47 blockade (Figure 4E). FACS analysis showed increased phagocytosis of labeled tumor cells by TAMs in 4D5-IgG2A-treated animals (Figure 4F), directly demonstrating 

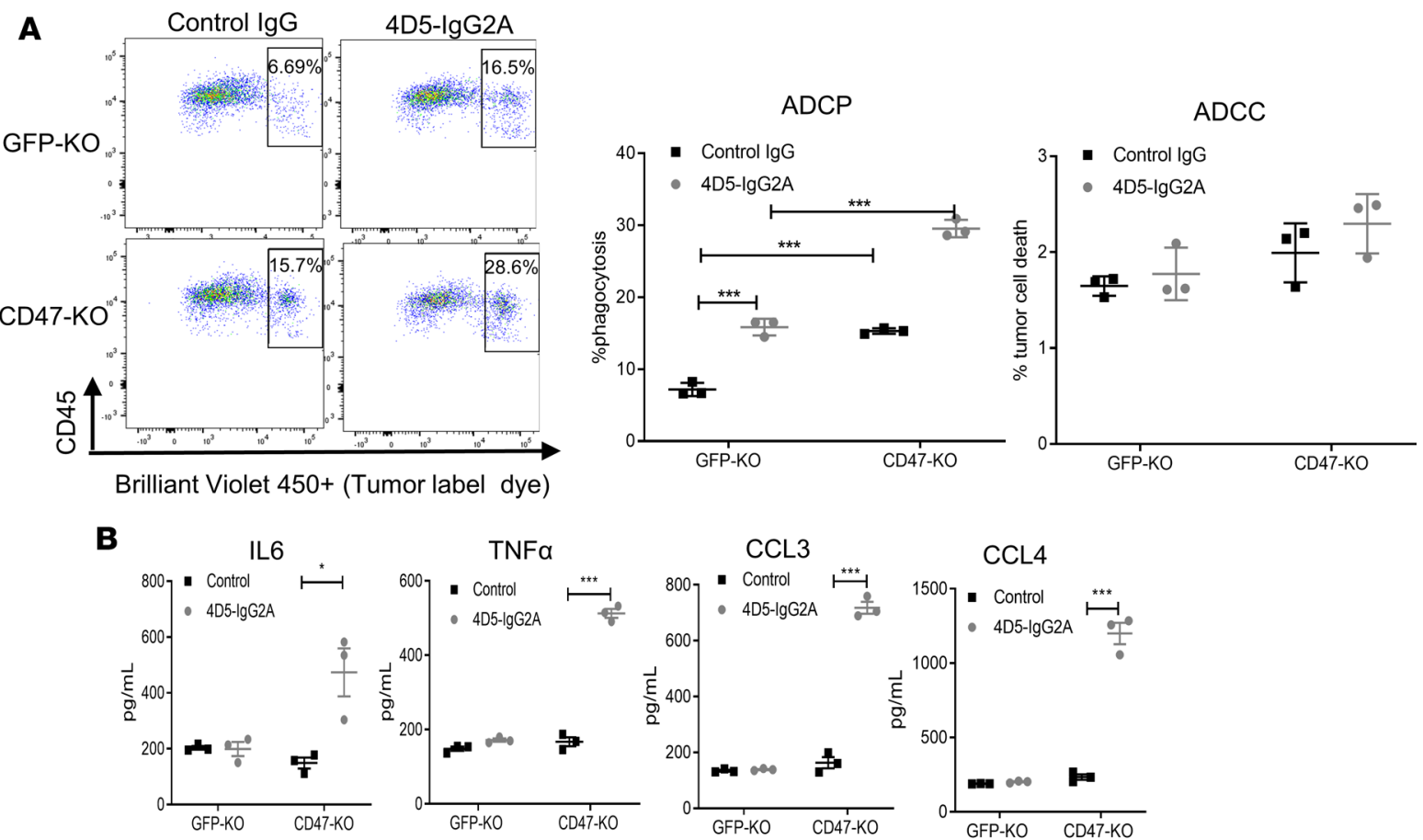

C

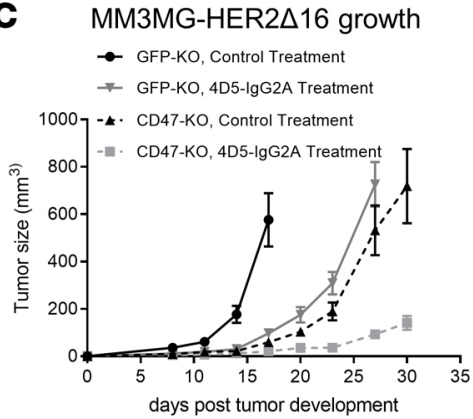

D

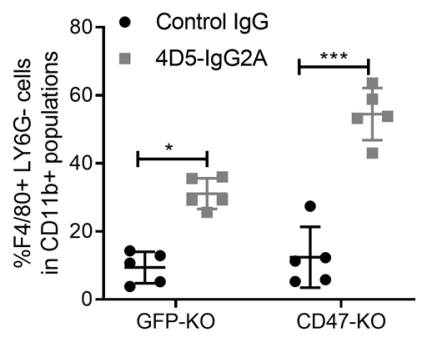

E Parental MM3MG-HER2 16

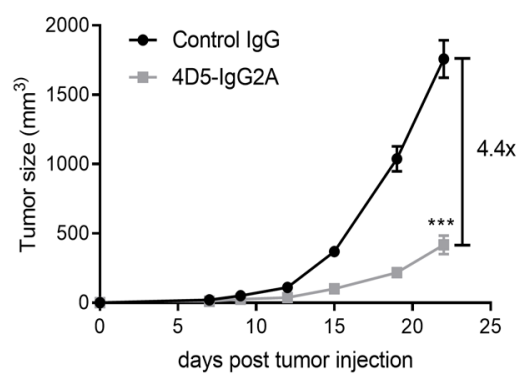

MM3MG-HER2 $\triangle 16$ CD47-OE

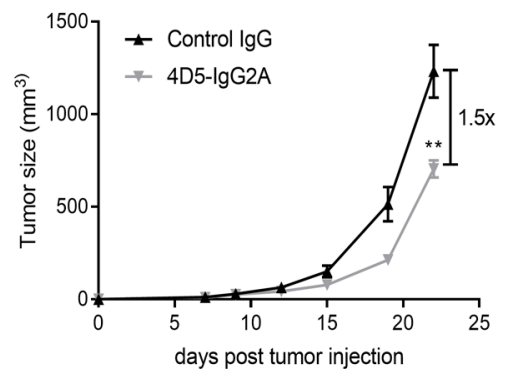

$\mathbf{F}$

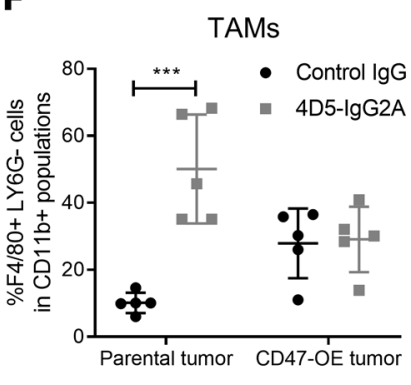

Figure 3. CD47 suppresses the antitumor activity of mouse trastuzumab (4D5). (A) CD47-KO cells were generated from MM3MG-HER2 16 cells using CRISPR/Cas9 technology. A control GFP-KO line was generated in parallel. Control and CD47-KO MM3MG-HER2 16 cells were labeled with Brilliant Violet 450 Dye and incubated with bone marrow-derived macrophages (BMDMs) at a 3:1 ratio with control or 4D5 antibodies (10 $\mu \mathrm{g} / \mathrm{mL}$ ). Antibody-dependent cellular phagocytosis (ADCP) and cytotoxicity (ADCC) were measured as described in Figure $1 \mathrm{H}$. $n=3$. Experiment was repeated 2 separate times using CD47-KO clones containing a different guide RNA. (B) Cytokines and chemokines secreted by macrophages from coculture experiment with HER2 ${ }^{+} \mathrm{BC}$ were analyzed using the Luminex platform. Additional cytokines detected can be found in Supplemental Figure $5 . n=3$. (C and D) Control and CD47-KO MM3MG-HER2 16 cells were implanted into mouse mammary fat pads and treated with 4D5-IgG2A or control antibodies as described before (Figure 1B). TAMs were analyzed by FACS after tumor volume reached $>1000 \mathrm{~mm}^{3}$. $n=5$. (E and F) Cd47-overexpressing cells (CD47-OE) were generated in MM3MG-HER2 $\triangle 16$ cells after transduction with Cd47 cDNA under the control of the EF1s promoter. CD47-0E tumor cell growth was compared to parental MM3MG-HER2 16 cells in mice treated with control antibody or 4D5-IgG2A. TAMs were analyzed by FACS. $n=5$. Significance was determined by 1-way ANOVA with Tukey's multiple-comparisons test (A, B, D, and F) or 2-way ANOVA with Tukey's multiple-comparisons test (C and $\mathbf{E})$. All data represent the mean $\pm \mathrm{SEM} .{ }^{*} P<0.05 ;{ }^{*} P<0.01 ;{ }^{* *} P<0.001$. 
that 4D5-IgG2A treatment promotes ADCP of HER2 ${ }^{+}$tumor cells in vivo. Furthermore, we found that the addition of CD47 blockade further increased ADCP of labeled tumor cells by TAMs (Figure 4F). As expected, this therapeutic mechanism requires the engagement with FCGRs on macrophages, since ADCP of tumor cells induced with 4D5 plus anti-CD47 in vivo was completely abolished in Fcerlg-KO mice (Figure 4G). In sum, these studies demonstrate that HER2 mAb stimulates ADCP from endogenous TAMs against HER2 ${ }^{+} \mathrm{BC}$, which can be boosted via combination with CD47 blockade therapy.

CD47 blockade synergizes $4 D 5$ therapeutic activity in a transgenic HER $2^{+} B C$ mouse model. Having demonstrated efficacy in an orthotopic model of HER2 ${ }^{+} \mathrm{BC}$, we wanted to extend our study using a spontaneous model of HER2 ${ }^{+} \mathrm{BC}$ that approximates a late-stage HER2 ${ }^{+} \mathrm{BC}$ (where HER2 mAbs are not highly effective) (43). Analogous to a clinical trial (Figure 5A), the individual animals with palpable breast tumors $\left(\sim 200 \mathrm{~mm}^{3}\right)$ were enrolled in a specific treatment group. We found that mice in the $4 \mathrm{D} 5$-IgG2A monotherapy treatment group had a significant increase in survival time and delayed tumor growth, whereas CD47 blockade monotherapy had no significant effect compared to the control group (Figure 5, B and C). Strikingly, combination therapy of 4D5-IgG2A with CD47 blockade resulted in a further prolonged survival rate and delayed tumor growth compared with 4D5 monotherapy, suggesting that this combination may be efficacious in advanced HER2 ${ }^{+}$BCs. To determine if these therapies again alter the immune infiltrates, we analyzed the composition of the tumor microenvironment by flow cytometry. As before, we found an increase in TAMs within the 4D5-IgG2A monotherapy group, whereas the combination therapy group showed an even higher increase (Figure 5D). Additionally, we also observed a slight reduction in T cell infiltration and neutrophil levels (Supplemental Figure 6B).

Single-cell transcriptome analysis of TAMs in HER2 $2^{+}$BC after 4D5 with CD47 blockade combination therapy. To further determine if macrophages were differentially activated, we performed single-cell RNA sequencing on dissociated tumors from the HER2-transgenic mice. These studies confirmed the increase in macrophages upon 4D5-IgG2A plus anti-CD47 treatment and also revealed the emergence of a distinct group of macrophages (that we termed "Phag $\mathrm{M} \Phi$ " cluster) that are phenotypically distinct from the resident macrophage clusters (i.e., "M1 M $\Phi$ " and "M2 M $\Phi$ " clusters) (Figure 6, A and B). Notably, we found that this Phag MФ cluster contained large quantities of human HER2 RNA and other tumor-specific transcripts (such as Epcam and cytokeratins), indicating that they have actively phagocytosed tumor cells. This cluster was expanded by 4D5-IgG2A treatment and increased further by combination 4D5 plus CD47 mAb treatment (Figure 6B and Table 1). In agreement with our FACS analysis, the levels of total macrophages were significantly increased, while $\mathrm{T}$ cell and neutrophil levels were reduced, after 4D5 or combination therapy (Figure 6B and Table 1). Interestingly, the frequency of cytotoxic gene expression (Ifng and $G z m b$ ) among $\mathrm{CD}^{+} \mathrm{T}$ cells was increased following treatments (Figure 6 and Supplemental Table 1).

Using differential gene expression analysis, we first assessed the impact of our treatments on the M1-like and M2-like macrophage clusters in comparison to control (Figure 7, A and B). Of note, these 2 macrophage clusters do not demonstrate evidence for hyperphagocytosis of tumor cells at this time point of analysis, as evidenced by their lack of tumor marker uptake (Figure 6B). Gene expression data revealed that our treatments promoted macrophage polarization into a proinflammatory antitumor phenotype, as evidenced by an increase in genes involved in interferon, inflammatory cytokine, chemokine, and TLR pathways (Figure 7, A and B). Accordingly, these changes were the most significant with combination therapy, and also more strongly observed in the M1-like MФ cluster.

In contrast, the Phag M cluster (predominantly present in the combination treatment group) had surprisingly increased expression of gene signatures for wound repair (e.g., thrombospondins and tenascins), ECM remodeling (e.g., collagens and MMPs), growth factors (e.g. Igfl, Tgfb, and Egf), and antiinflammatory genes (e.g., IL4, IL13, and $I L 1 r$ ) compared with the other $2 \mathrm{M} \Phi$ clusters (Figure 7C). This was also accompanied by decreased expression of genes for proinflammatory cytokines/chemokines, phagocytosis/ opsonization, and antigen presentation (Figure 7C).

These single-cell RNA sequencing analyses revealed that while trastuzumab with CD47 blockade polarizes macrophages into an antitumor phenotype and greatly increases tumor phagocytosis, prolonged treatment and continuous tumor hyperphagocytosis may also trigger a transcriptional switch in TAMs for repair of ADCP-induced tissue damage. Thus, while these studies demonstrate the antitumor efficacy of trastuzumab plus CD47 blockade, they also suggest that prolonging this process can trigger a wound healing response in macrophages that could have protumor and/or immunosuppressive functions (44-46). 
A MM3MG-HER2 $\triangle 16$ growth

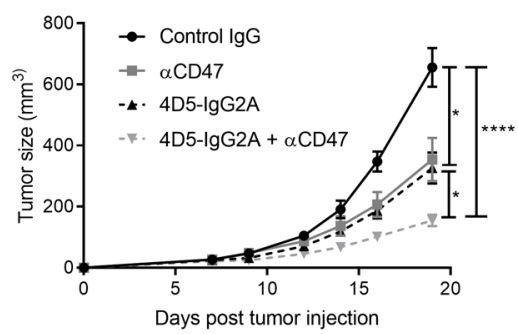

B

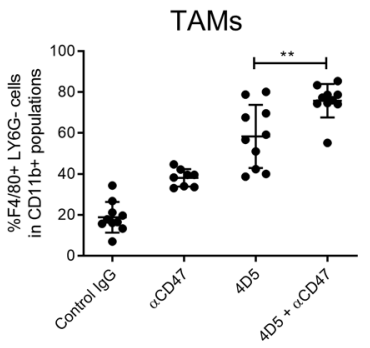

C MM3MG-HER2 $\triangle 16$ growth In SCID-Beige

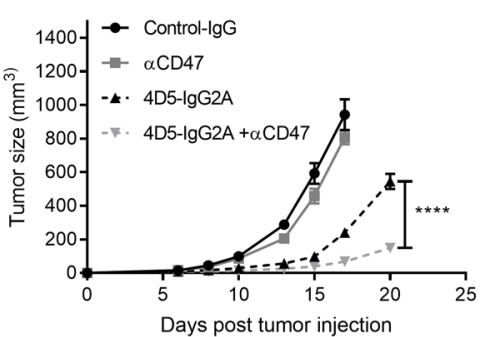

D

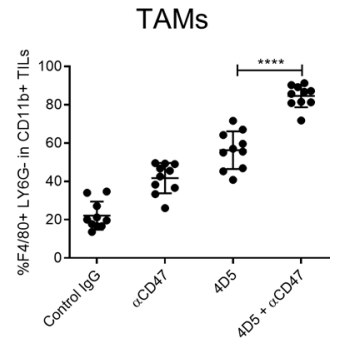

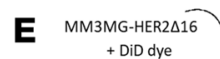
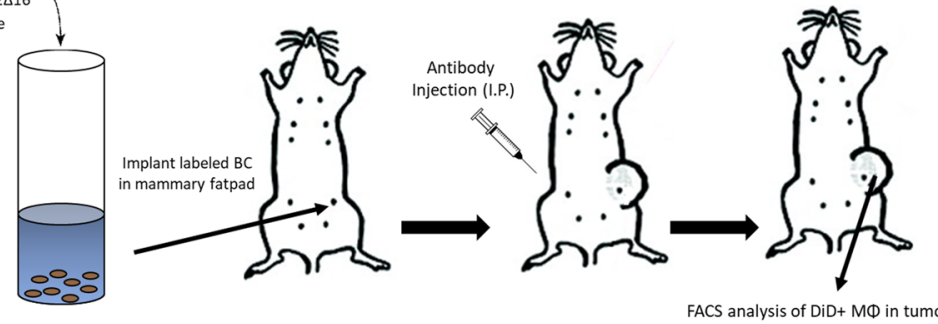

F

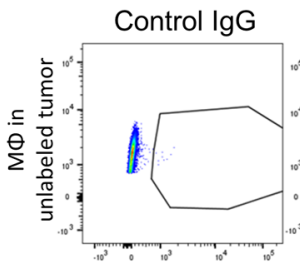

4D5-IgG2A

4D5-lgG2A + aCD47

FACS analysis of DiD

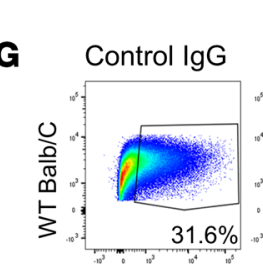

4D5- $\lg$ G2A
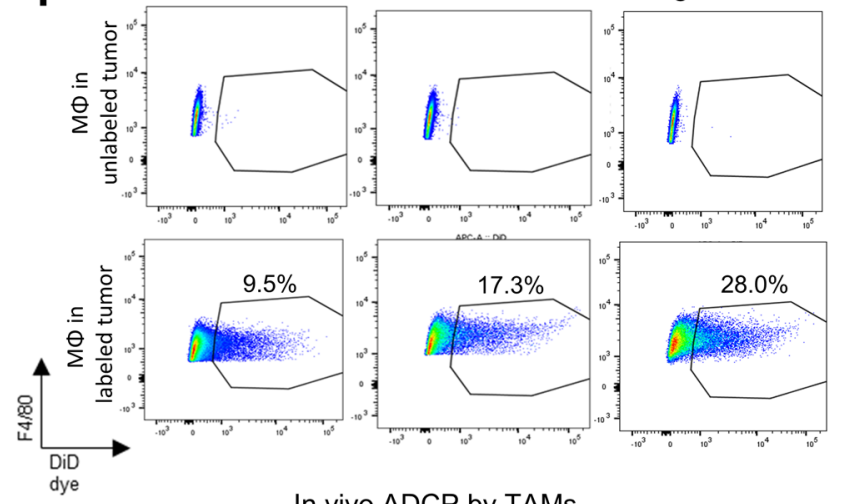

In vivo ADCP by TAMs

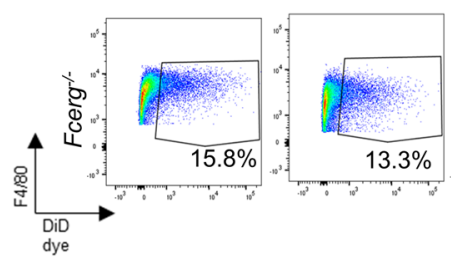

In vivo ADCP by TAMs
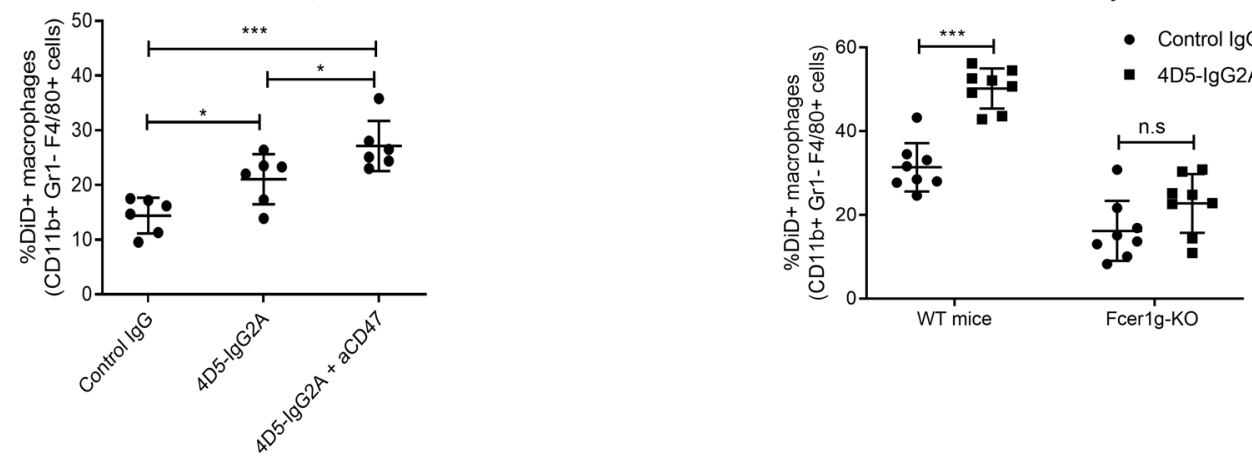

Figure 4. CD47 blockade increases therapeutic efficacy of mouse trastuzumab and augments tumor-associated macrophage (TAM) expansion and phagocytosis. (A) Tumor growth experiment (as in Figure 1B) was repeated using CD47 blockade antibody (MIAP410, $300 \mu g$ per mouse) alone or in combination with 4D5-IgG2a. (B) TAM populations were analyzed by FACS after tumor volume reached $>1000 \mathrm{~mm}^{3}$. Analysis of additional immune cell types are shown in Supplemental Figure 4D. Data represent the mean \pm SEM. $n=8-10$. (C) Repeat of similar tumor growth experiment and treatments in SCID-beige mice. (D) TAM populations from SCID-beige experiment were analyzed by FACS. $n=10$. (E) Schematic representation of in vivo antibody-dependent cellular phagocytosis (ADCP) experiment. MM3MG-HER2 $\triangle 16$ cells were labeled with Vybrant DiD dye and implanted $\left(1 \times 10^{6}\right.$ cells) into mammary fat pads of BALB/c mice. Once tumor volume reached approximately $1000 \mathrm{~mm}^{3}$, mice were treated with control antibody, 4D5-IgG2A (200 $\mu \mathrm{g})$, or 4D5-IgC2A in combination with MIAP410 (300 $\mu \mathrm{g})$. On the next day, tumors were harvested and tumor-phagocytic macrophages were quantified by FACS. (F) Representative FACS plots and graphical summary showing frequency of macrophages (CD11b+F4/80+LY6C- $\mathrm{LY}^{+} 6 \mathrm{C}^{-}$) that have phagocytosed DiD-labeled tumor cells. $n=6$. (C) A similar in vivo ADCP experiment was repeated in Fcer1 $g^{-1-}$ mice. $n=8$. Significance was determined by 2-way ANOVA with Tukey's multiple-comparisons test (A and $\mathbf{C}$ ) or 1-way ANOVA with Tukey's multiple-comparisons test (B, D, F, and $\mathbf{G})$. All data represent the mean \pm SEM. ${ }^{*} P<0.05,{ }^{* *} P<0.01,{ }^{* *} P<0.001,{ }^{* * * *} P<0.0001$. 
A

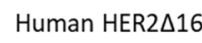

transgenic mice

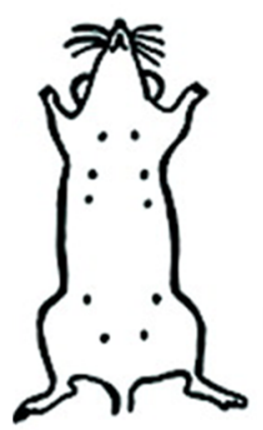

Detect palpable tumors

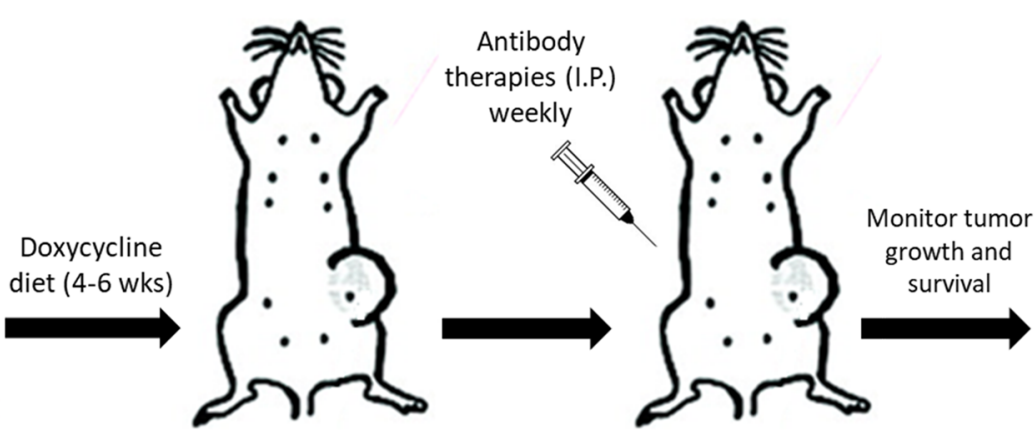

B Transgenic HER2 $\triangle 16 \mathrm{BC}$ mouse model Survival

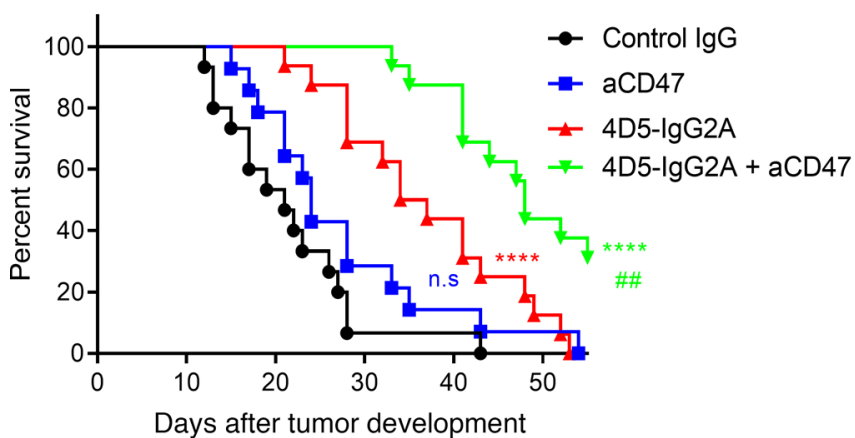

C

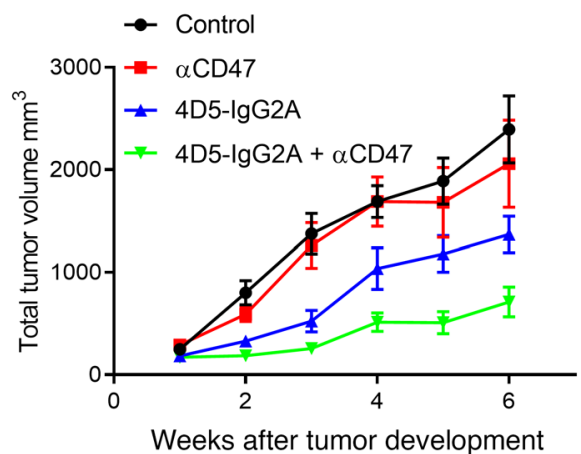

D

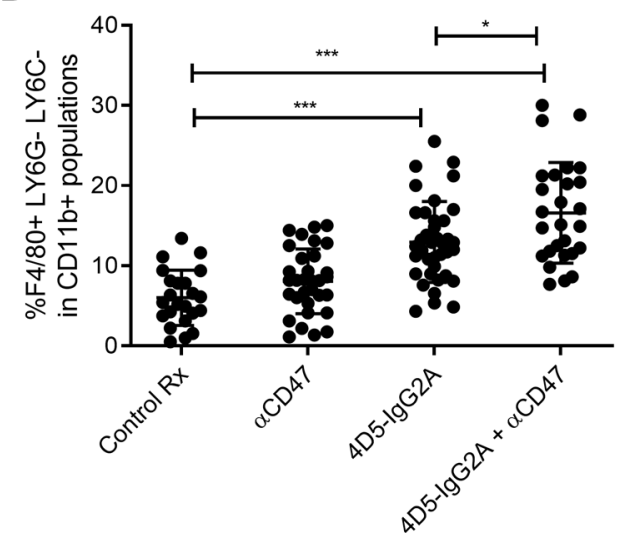

Figure 5. CD47 blockade synergizes with mouse trastuzumab therapeutic activity in a transgenic human HER2+ breast cancer (BC) mouse model. (A) Schematic representation of experiment using the endogenous human HER2-transgenic mouse model. Spontaneous breast tumors in the transgenic animals were induced with doxycycline diet. Four treatment arms were set up: control IgG (200 $\mu$ g weekly, $n=15)$; CD47 blockade (MIAP410, $300 \mu$ g weekly, $n=14$ ); 4D5-IgG2A (200 $\mu$ g weekly, $n=16)$; and 4D5-IgG2A combined with MIAP410 $(n=16)$. Individual animals were consecutively enrolled into a specific treatment arm as soon as palpable breast tumors were detected $\left(\sim 200 \mathrm{~mm}^{3}\right)$. (B) Survival of mice in each treatment arm; time of start is on the day of palpable tumor detection and treatment enrollment. Log-rank (Mantel-Cox) test for survival analysis: ${ }^{* * *} P<0.0001$ for treatment vs. control group; ${ }^{\# \# P} P 0.01$ for difference observed between 4D5 group and 4D5 plus $\alpha$ CD47 group. (C) Tumor burden in animals from each treatment arm was measured over time after enrollment in treatment arm. Each animal developed 1-4 total tumors in their mammary fat pads. The total tumor burden per mouse is shown. Animals were euthanized when their total tumor volume reached more than $2000 \mathrm{~mm}^{3}$. (D) Tumors in the transgenic mice were harvested, processed into single-cell suspensions, and analyzed by FACS. Each tumor was treated as an individual measurement. Data are shown as the mean \pm SEM. Control IgG $n=23, \alpha \operatorname{CD} 47 n=27,4 \mathrm{D} 5 n=38,4 \mathrm{D} 5+\alpha \mathrm{CD} 47 n=32 .{ }^{*} P<0.05$, ${ }^{* * *} P<0.001$ by 1 -way ANOVA with Tukey's multiple-comparisons test.

Human CD47 gene expression is a prognostic factor in $\mathrm{HER}^{+} \mathrm{BC}$ and limits the therapeutic activity of trastuzumab. As all of our investigations had been performed in different murine HER2+ BC models, we also wanted to determine if ADCP activity of trastuzumab can be seen in human HER2 ${ }^{+} \mathrm{BC}$ and if CD47 could likewise limit its antitumor efficacy. Based on our findings, we hypothesized that $C D 47$ expression may allow for resistance and reduced survival of HER2 ${ }^{+}$BC patients undergoing trastuzumab therapies. To investigate this hypothesis, we utilized the Molecular Taxonomy of Breast Cancer International Consortium (METABRIC) gene expression data set (47) and stratified BC patients of different molecular subtypes into CD47-high and CD47-low groups based on optimum threshold. This analysis revealed that $C D 47$ gene expression associates with lower patient overall survival (Figure 8A) and was most significant in the HER2 ${ }^{+}$molecular subtype compared with triple-negative $\mathrm{BC}$ or $\mathrm{ER}^{+}$ subtypes (Figure $8 \mathrm{~B}$ ). This suggests that CD47 signaling may be an important resistance mechanism for HER2 ${ }^{+} \mathrm{BC}$ and trastuzumab therapy. 
A
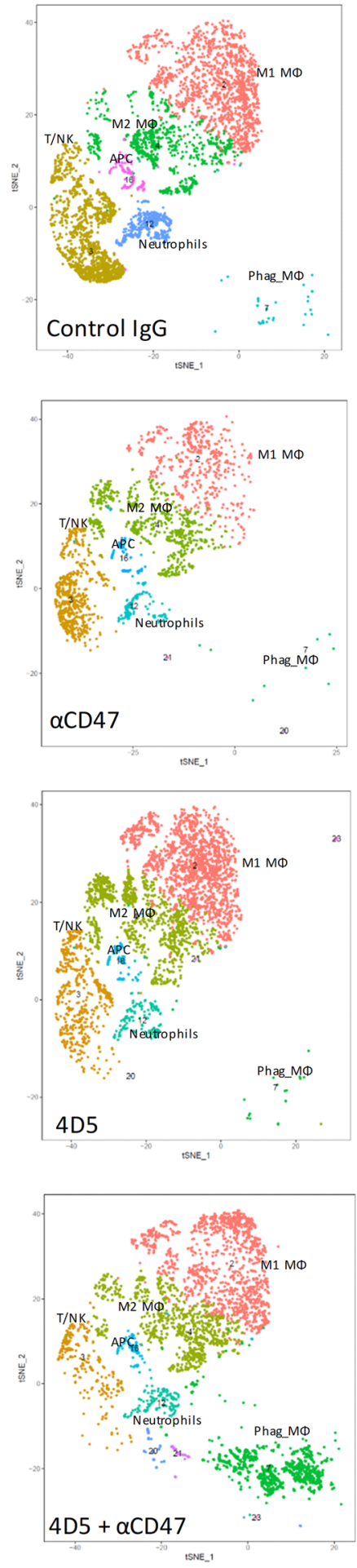

B

\section{Control IgG}

Gene Category

M1-like MФ

M2-like M $\Phi$ Neutr

CD8/NK

CD4 APC
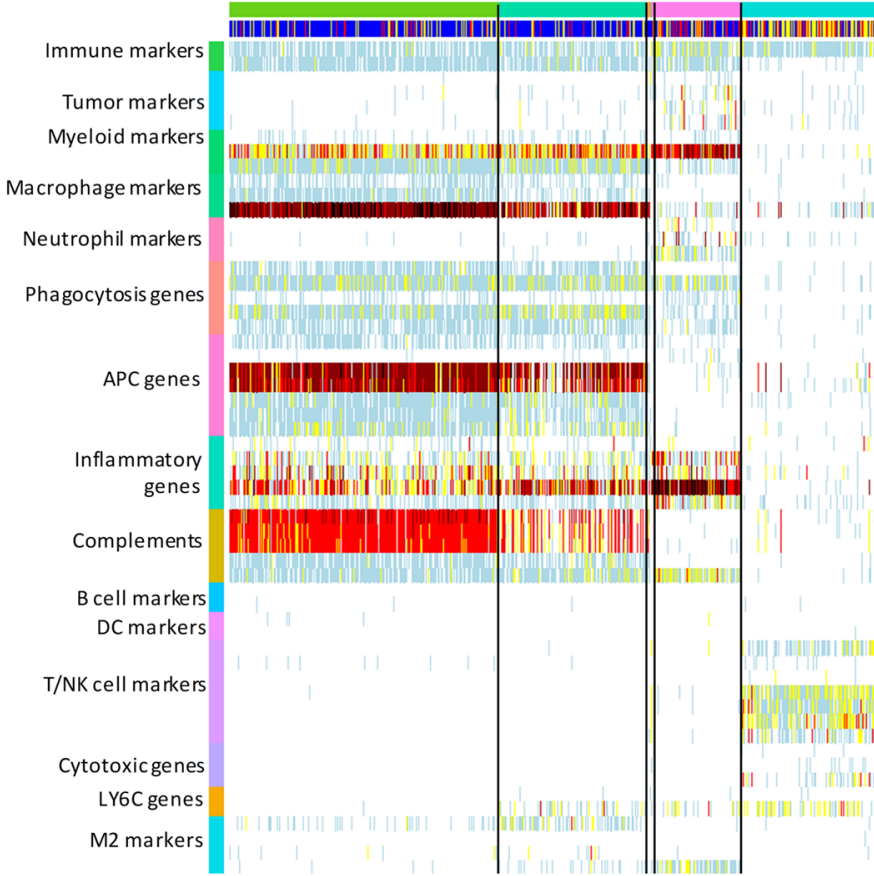

|||||||||||| |||||||||||| || || ||||||||||| Condition CCPhase

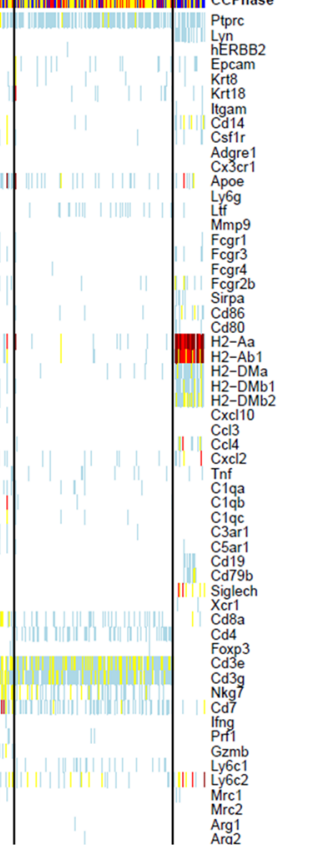

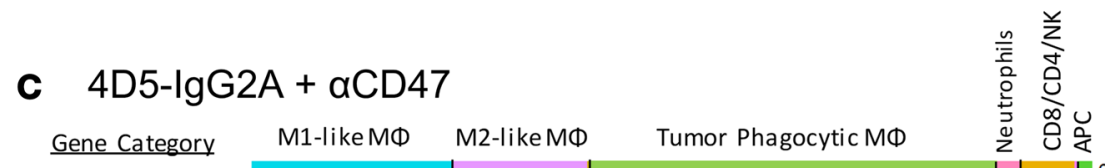

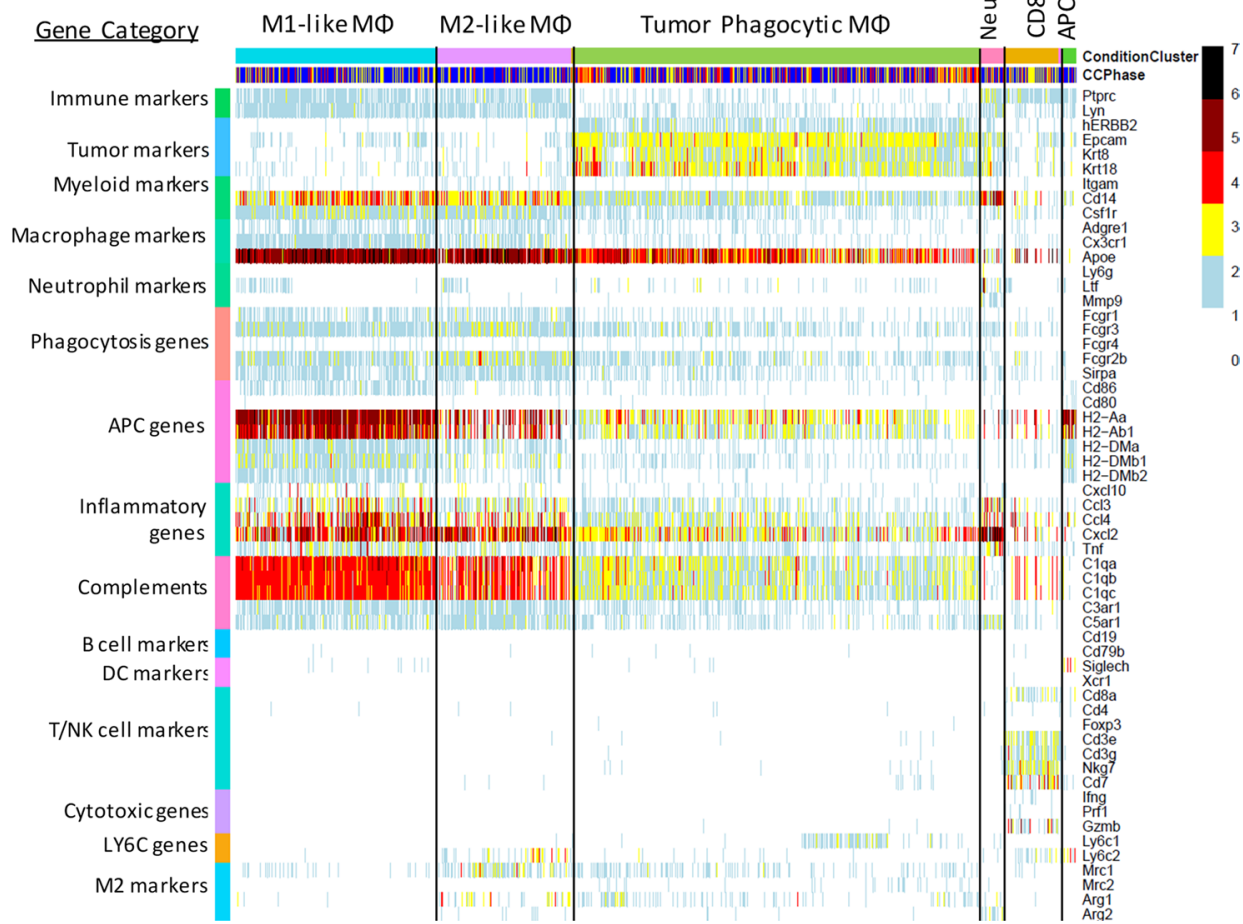

Figure 6. Single-cell transcriptome analysis of immune clusters within HER2 ${ }^{+}$breast cancer after trastuzumab with CD47 blockade therapy. HER2 ${ }^{+}$ tumors from HER2 $\triangle 16$-transgenic animals were isolated for single-cell RNA sequencing using the $10 \times$ Genomics platform. Data from all tumors were pooled for clustering and gene expression analysis. (A) tSNE plots showing distinct clusters of immune cells in tumors from 4 treatment groups: control IgG, $\alpha$ CD47, 4D5-IgG2A, or combination. (B and C) Heatmap of relevant gene markers confirmed the various immune cell clusters in control tumors (B) and the expansion of macrophage clusters in the combination therapy-treated tumors (C). Macrophages that contained tumor-specific transcripts (e.g., hERBB2, Epcam, Krt8) were categorized as tumor-phagocytic macrophages (Phag MФ, predominantly found in the combination treatment group). 
Table 1. Single-cell transcriptome analysis of macrophage cluster sizes and frequency of tumor-phagocytic macrophages

\begin{tabular}{|c|c|c|c|c|}
\hline Treatment & No. of tumors analyzed & $\begin{array}{l}\text { Average length on } \\
\text { treatment regimen }\end{array}$ & $\begin{array}{c}\text { МФ cluster size } \\
\text { (\% MФ among immune cells) }\end{array}$ & 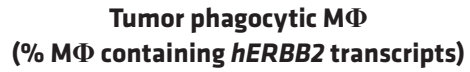 \\
\hline Control & 3 & 28 days & 2354 of $4527(52 \%)$ & $4.22 \%$ \\
\hline$\alpha C D 47$ & 2 & 36 days & 1228 of 2154 (57\%) & $3.95 \%$ \\
\hline 4D5-IgC2A & 4 & 45 days & 2938 of 3815 (77\%) & $9.07 \%$ \\
\hline 4D5-IgG2A + $\alpha$ CD47 & 4 & 56 days & 4673 of 5079 (92\%) & $48.44 \%$ \\
\hline
\end{tabular}

Numbers represent the mean of replicates in each treatment group.

We next investigated whether human CD47 limits the ADCP effect of trastuzumab against amplified HER2+ human BC cells. To address this in vitro, we first generated CD47-KO KPL-4 (HER2+ BC) cells (Supplemental Figure 4C) and compared them to controls after trastuzumab treatment in ADCP experiments using human PBMC-derived macrophages. As in mouse studies, we found that loss of human CD47 in tumor cells increased their susceptibility to ADCP elicited by trastuzumab (Figure 8C). To determine if this antitumor effect also occurs in vivo against human HER2 ${ }^{+}$BC cells, we implanted KPL-4 control and CD47-KO cell lines into SCID-beige mice (which contain a mouse SIRP $\alpha$ that can bind to human CD47; see ref. 48) and treated them with clinical-grade trastuzumab. As before, we saw a strong effect from trastuzumab treatment that was significantly enhanced with CD47-KO, resulting in tumors being completely eliminated (Figure 8D). In trastuzumab-treated mice, we again found a significant increase in TAMs (Figure $8 \mathrm{E}$ ) and an upregulation of proinflammatory genes (Figure $8 \mathrm{~F}$ ), as seen in the murine tumor model. Unfortunately, the complete regression of tumors treated with CD47-KO plus trastuzumab precluded any further analysis of these tumors. Collectively, these studies suggest that the dominant antitumor mechanism of trastuzumab therapy is through ADCP of HER2 ${ }^{+}$tumor cells, which can be substantially impaired through the CD47/SIRP $\alpha$ axis. This suggests that combinatorial therapy with CD47 blockade could be beneficial in patients with trastuzumab resistance.

\section{Discussion}

Even since the demonstration of clinical benefit provided by therapeutic HER2-specific mAbs to patients with HER2-overexpressing BC, the mechanism of action for the therapeutic HER2 mAb trastuzumab has been the subject of numerous studies. Some reports suggest that trastuzumab may both block oncogenic HER2 signaling and induce $\operatorname{ADCC}(7,49,50)$. Using murine versions of the clinically approved HER2-specific mAb trastuzumab, our in vitro studies confirmed these reported MOAs, specifically blockade of HER2 signaling and trastuzumab-mediated ADCC by NK cells. In contrast, the in vivo antitumor mechanisms of trastuzum$\mathrm{ab} / 4 \mathrm{D} 5$ remain less conclusive, with early studies suggesting the importance of signal blockade $(51,52)$ and subsequent studies demonstrating the direct involvement of ADCC eliciting FcR-expressing cells (10) (such as neutrophils and NK cells), and more recent studies highlighting the importance of adaptive immunity $(8,15)$. Notably, few studies have examined trastuzumab-mediated ADCP, with a single study documenting the ability of trastuzumab to elicit ADCP in vivo (16), while another study suggested that trastuzumab-mediated ADCP from TAMs is immunosuppressive (17). Consequently, our models and agents provide a reliable platform and opportunity to interrogate the in vivo antitumor mechanism of HER2-specific mAbs against HER2-driven BC.

In this study using multiple models of human HER2-expressing BC, i.e., MM3MG-HER2 16 , KPL-4, and an endogenous transgenic HER2 ${ }^{+} \mathrm{BC}$ model that is tolerant to human HER2, and using the murine version of trastuzumab with the functionally equivalent mouse isotype (4D5-IgG2A), we demonstrate that macrophages are the major effectors carrying out the antitumor immunity of trastuzumab therapy through ADCP. Although TAMs have been shown to promote tumor progression, it is known that they also retain their $\mathrm{Fc}$-dependent antitumor function when induced by targeted therapies (i.e., mAbs) (53, 54). Our conclusion about the therapeutic impact of TAMs is supported by the following findings: (a) the therapeutic effect of trastuzumab is equivalent in wild-type and in SCID-beige mice and does not alter systemic HER2-specific adaptive immunity and T cell/NK cell infiltration in tumors, indicating that adaptive immunity and NK cells are not necessary immune cells to mediate antitumor effects; (b) the depletion of macrophages but not neutrophils had a significant negative effect on trastuzumab efficacy; (c) trastuzumab 
A Activation of M1-like TAM
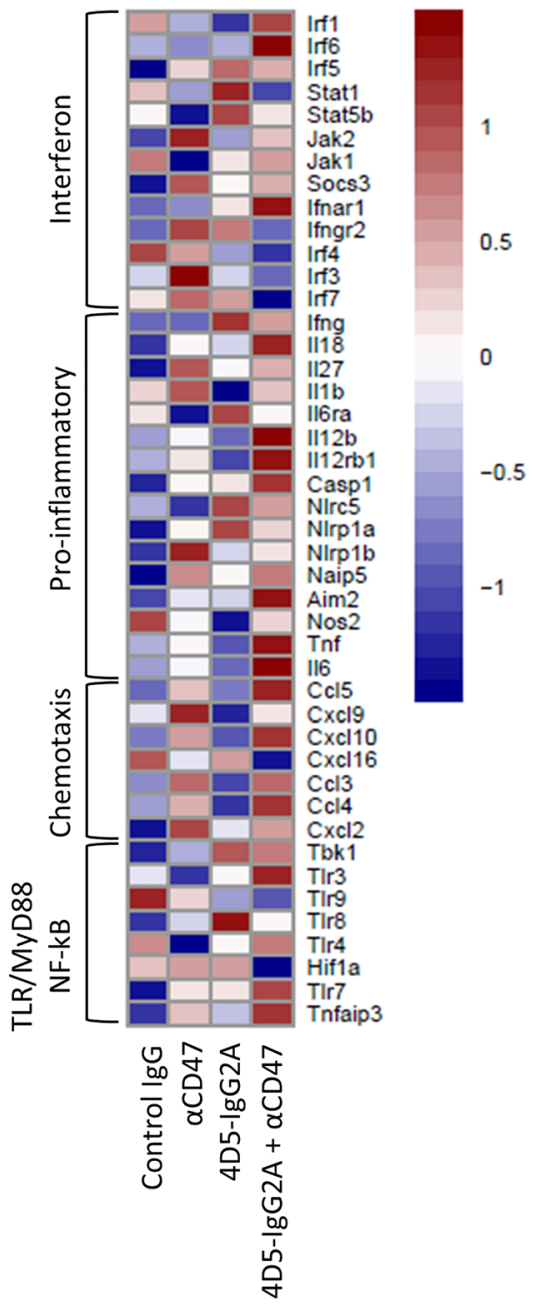

B Activation of M2-like TAM
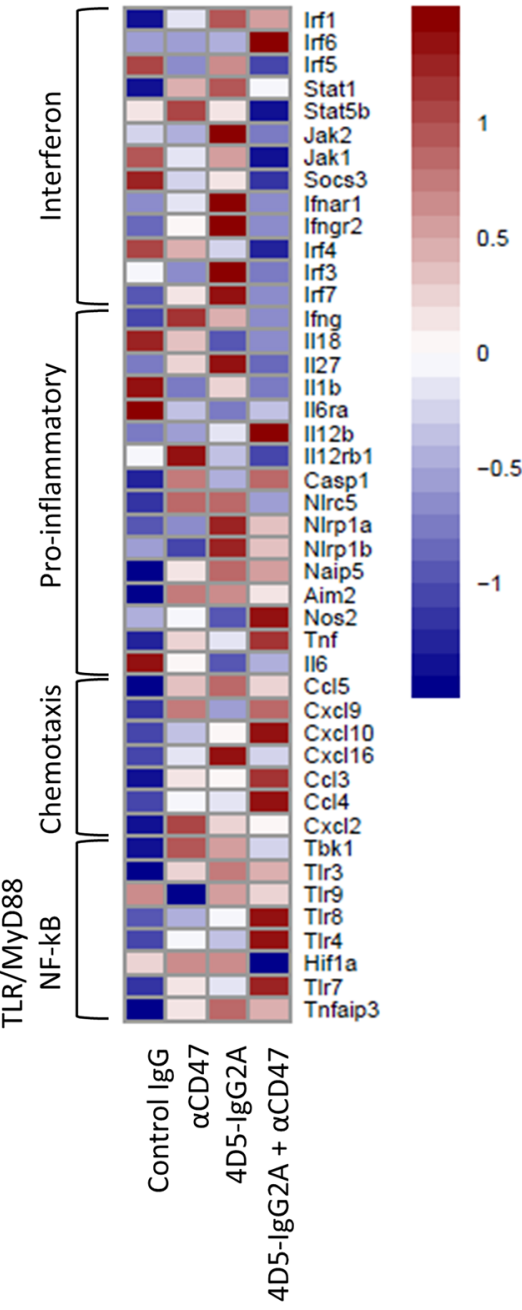

C

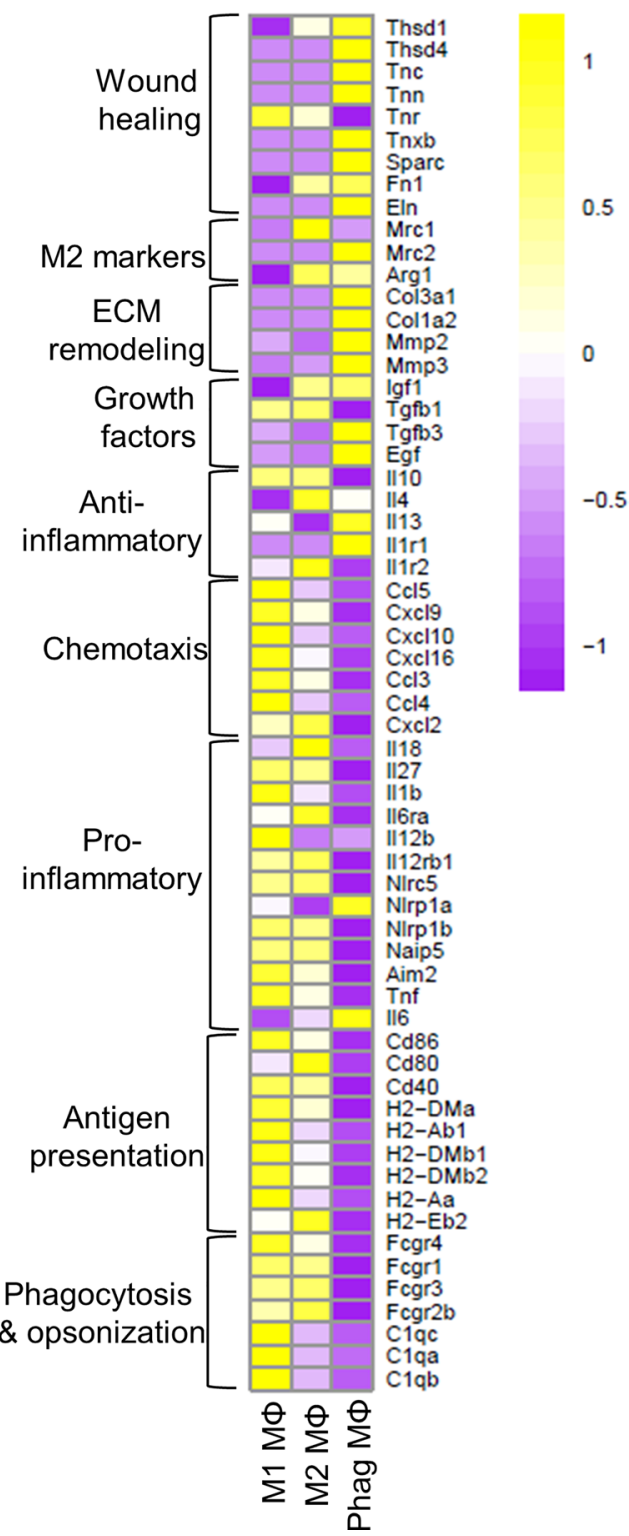

Figure 7. Differential gene expression analysis of TAM clusters in HER2+ breast cancer after trastuzumab with CD47 blockade therapy. (A and B) Differential gene expression analysis of gene signatures for IFN, proinflammation, chemotaxis, and TLR/MyD88/NF- $\kappa B$ pathways in M1-like M $\Phi$ clusters (A) and M2-like MФ clusters (B) revealed how they were affected by the treatment regimens. (C) Differential gene expression analysis of immunoregulatory gene signatures (wound healing, ECM remodeling, growth factors, antiinflammation) versus immunostimulatory gene signatures (proinflammation, chemotaxis, antigen presentation, phagocytosis/opsonization) among the 3 distinct macrophage clusters in the combined data set.

treatment greatly and consistently increased TAM frequency; (d) trastuzumab treatment induced ADCP of HER2 ${ }^{+}$tumor cells in vitro and in vivo in an $\mathrm{FC}_{\mathrm{C}}$ receptor-dependent fashion; (e) blocking of the innate immune ADCP CD47/SIRP $\alpha$ regulatory axis significantly enhanced trastuzumab therapeutic outcomes and also increased ADCP of tumor cells; (f) trastuzumab combination with CD47 blockade induced TAMs into a highly phagocytic, immunostimulatory, and antitumor phenotype but also produced a wound-healing, immunoregulatory group of TAMs after prolonged tumor phagocytosis.

Our study provides insight into the potential of utilizing TAMs as a potent mediator of innate antitumor immunity that can be further exploited. It was initially believed that macrophages were present in high numbers in solid tumors as a mechanism of rejection. However, it soon became clear that TAMs are typically unable to induce an effective antitumor response in the immunosuppressive tumor microenvironment (55). Furthermore, high TAM infiltration levels are often associated with poor patient prognosis in breast, lung, 
A

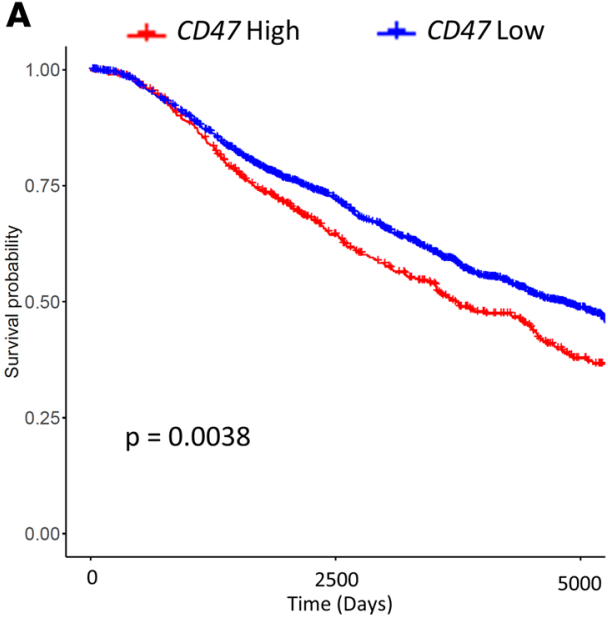

B

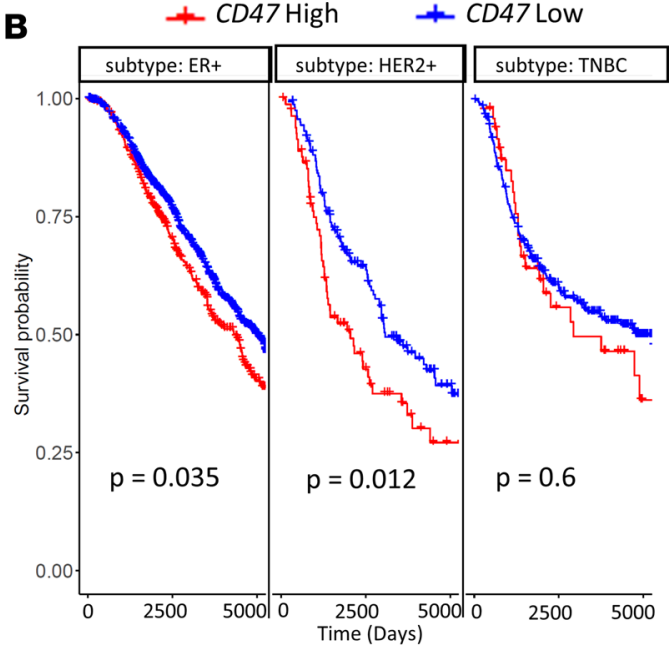

Human MDM phagocytosis of KPL-4 cells

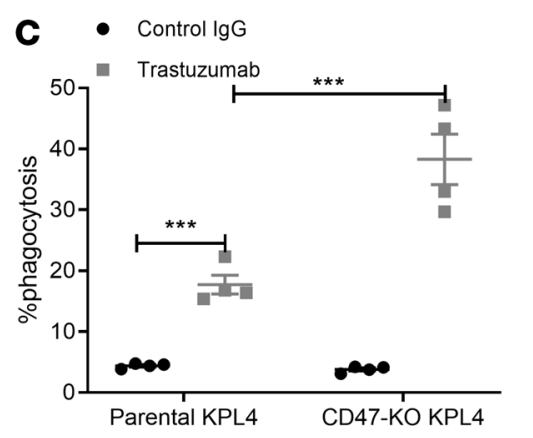

D

\section{KPL-4 growth in SCID-Beige}
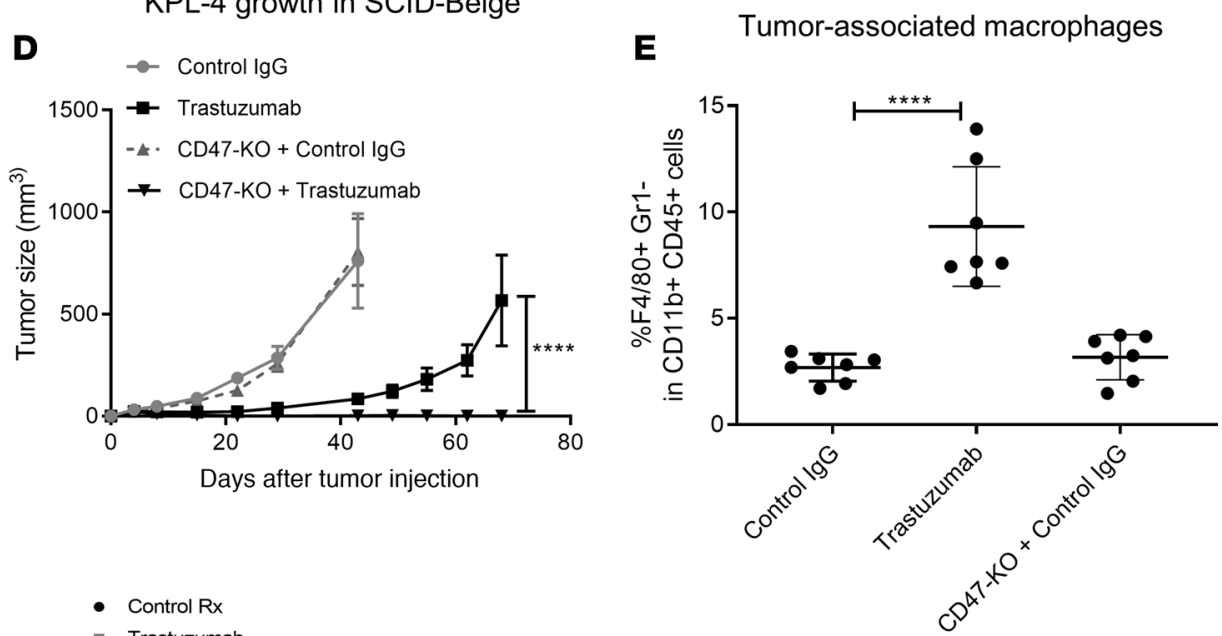

$\mathbf{F}$

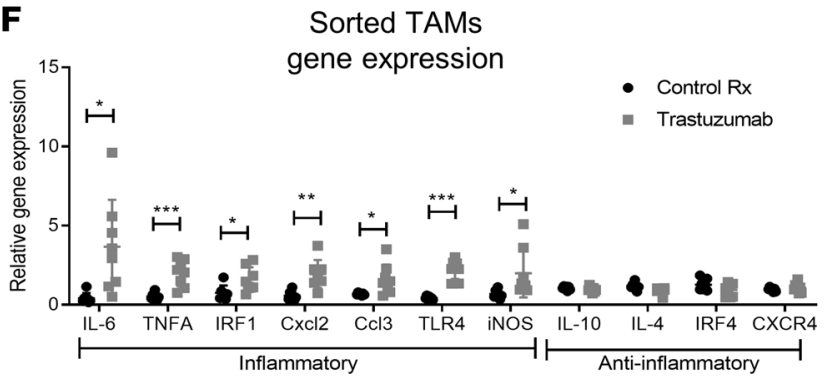

Figure 8. Human $C D 47$ gene expression is a prognostic factor in HER2 ${ }^{+}$breast cancer and limits the therapeutic activity of trastuzumab. (A and B) Kaplan-Meier survival curve for breast cancer (BC) patients' METABRIC data set. (A) Stratified into low and high groups based on average expression of $C D 47$ in all patients. (B) The same patient stratification based on disease subtype (ER+, HER2', and triple-negative BC [TNBC]). (C) CD47 knockout in human HER2+ BC line KPL-4 was generated using the CRISPR/Cas9 approach. Control and CD47-KO KPL-4 cells were labeled with Brilliant Violet 450 Dye, and incubated with human monocyte-derived macrophages (hMDMs) at a 3:1 ratio, in the presence of control lgG or trastuzumab (10 $\mu \mathrm{g} / \mathrm{mL}$ ). Antibody-dependent cellular phagocytosis (ADCP) was measured by percentage of hMDM uptake of labeled KPL-4 cells (CD45 and BV450+). Data are shown as the mean $\pm \mathrm{SEM} ; n=4$ biological replicates. Experiment was repeated using hMDMs generated from 3 healthy PBMC donors. (D) Control or CD47-KO KPL-4 cells were implanted into mammary fat pads of SCID-beige BALB/c mice $\left(5 \times 10^{5}\right.$ cells $)$. Trastuzumab $(50 \mu \mathrm{g})$ or control human IgG1 was administered weekly and tumor volume was measured. ${ }^{* * *} P<0.0001$ by 2 -way ANOVA with Tukey's multiple-comparisons test. (E) Tumor-infiltrating macrophage $\left(\mathrm{F} 4 / 80^{+} \mathrm{Cr} 1^{-} \mathrm{CD} 11 \mathrm{~b}^{+}\right)$populations were analyzed by FACS, except for the CD47-KO plus trastuzumab group, as no tumor growth occurred. Data are shown as the mean \pm SEM. $n=7$. (F) Tumor-associated macrophages from control-treated and trastuzumab-treated tumors were sorted by FACS (F4/80+Cr1-CD$11 \mathrm{~b}^{+} \mathrm{CD} 45^{+}$) and analyzed with RT-qPCR for the expression of pro- and antiinflammatory genes. Data are shown as the mean \pm SEM. $n=7$. Multiple 2 -sided $t$ test. In C and E, significance was determined by 1-way ANOVA with Tukey's multiple-comparisons test. ${ }^{*} P<0.05 ;{ }^{* *} P<0.01 ;{ }^{* *} P<0.001$.

prostate, liver, thyroid, pancreas, kidney, and many other solid cancer malignancies (56). Indeed, studies have shown that immunosuppressive TAMs can support tumor development by promoting angiogenesis, tissue invasion, metastasis, and suppressing tumor attack by NK cells and cytotoxic T lymphocytes (57). In contrast, TAMs in colorectal cancer have a more activated, immunostimulatory phenotype and interestingly, high 
TAM density in colorectal cancer correlates with increased patient survival $(54,58)$. Nonetheless, TAMs in multiple histologic types of tumors retain their expression of FCGRs and increasing evidence suggests mAbs can phenotypically modify immunosuppressive TAMs toward an antitumor phenotype $(53,54,59)$. As such, the manipulation of TAMs, potentially through a tumor-targeting mAb (e.g., trastuzumab) or targeting of regulatory axis receptors (e.g., CD47/SIRP $\alpha$ ), are promising therapeutic approaches for multiple types of cancer.

While previous studies $(8,9)$ have documented the involvement of T cell immunity in mediating HER2 $\mathrm{mAb}$ efficacy, we were unable to detect a significant enhancement of adaptive $\mathrm{T}$ cell responses with trastuzumab monotherapy in either our orthotopic or HER2-tolerant endogenous models of HER2 ${ }^{+} \mathrm{BC}$. This may be due to the nature of our tumor models, the timing of our analysis, or the specific mAb utilized. In our immunocompetent in vivo studies, we utilized both murine and human HER2 mAbs similar to trastuzumab (isotypes with a high A/I ratio), as well as both human HER2-transformed cells and an endogenous mouse model of HER2 ${ }^{+}$BC. Previous studies $(8,9,17)$ have utilized rat neu-expressing ErbB2 models, non-HER2-transformed cells, and/or alternate antibody isotypes (mouse IgG1 with a low A/I ratio), which may account for a lack of ADCP activity and alteration of immunogenicity. Of note, a recent study using a 4D5 antibody containing a mouse IgG1 isotype reported that HER2 mAb-elicited macrophage ADCP is an immunosuppressive mechanism (17). Given that the mouse IgG1 subclass strongly activates inhibitory FCGR signaling on effector cells (low A/I ratio) and therefore is very different from trastuzumab (human IgG1, high A/I ratio) $(18,19,40)$, this emphasizes the need for using functionally equivalent mouse isotypes in translational studies to accurately model human antibody therapy. Nevertheless, clinical studies have demonstrated significant associations between adaptive immune responses and trastuzumab plus chemotherapy efficacy (60). Phagocytosis of tumor cells by macrophages has been documented to boost the priming of tumor-specific adaptive $\mathrm{CD}^{+}$and $\mathrm{CD} 8^{+} \mathrm{T}$ cells $(36,61)$, while different types of chemotherapy have been documented to enhance phagocytosis and augment immunogenic tumor cell death (62). Taken together, our results show that the clinical use of immunogenic chemotherapy combinations could stimulate adaptive immunity that would be potentially enhanced by trastuzumab-mediated ADCP. However, in the absence of strong immune stimulation (potentially through chemotherapy or immunogenic cell lines), trastuzumab does not appear highly effective at eliciting adaptive immunity and functions mainly through the stimulation of ADCP.

In identifying $\mathrm{ADCP}$ as a critical mechanism for trastuzumab efficacy, we also explored if it could be further enhanced through the blockade of the CD47 innate immune checkpoint. CD47 is highly expressed in BC and functions to suppress phagocytosis through binding with SIRP $\alpha$ on macrophages $(23,24)$. Interestingly, we found that CD47 gene expression is a negative prognostic factor in human BC, most significantly in HER2 ${ }^{+} \mathrm{BC}$. As treatment of HER2-overexpressing tumors with trastuzumab has been available for many years, this observation suggests that CD47 may be functioning in trastuzumab-treated patients to mediate $\mathrm{ADCP} /$ therapeutic resistance. This conclusion is supported by the enhanced effects observed between trastuzumab and CD47 blockade in augmenting ADCP and antitumor effects in our study. Moreover, single-cell transcriptome analysis of the tumor microenvironment demonstrates that trastuzumab therapy stimulates TAMs into a proinflammatory antitumor phenotype, which is further boosted by CD47 blockade (Figure 7, A and B). Such changes in macrophage phenotypes were also observed in cocultured ADCP experiments. This suggests that combination of targeted mAb therapy with CD47/SIRP $\alpha$ blockade could be beneficial in HER2 ${ }^{+}$BC and potentially other solid tumors. Proof-of-concept studies using tumor-targeting mAbs and CD47 blockade have been demonstrated in preclinical lymphoma models, as well as a recent phase I study of anti-CD20 mAbs (rituximab) and CD47 blockade, in rituximab-refractory non-Hodgkin lymphoma patients $(41,63)$.

Additionally, by implementing different methods such as multicolor FACS analysis and single-cell transcriptome analysis, we are the first to our knowledge to demonstrate in vivo tumor phagocytosis by macrophages upon combination of trastuzumab with CD47 blockade therapies. Moreover, we were able to identify a distinct cluster of hyperphagocytic TAMs within the TME. The identification of this population of TAMs may also serve as a predictive biomarker of this form of therapy. Gene expression analysis suggested that after profound phagocytosis of tumor cells, these macrophages switched to a tissue repair phenotype, as evidenced by their upregulation of gene signatures for wound healing, growth factors, ECM remodeling, and antiinflammatory markers compared with resident macrophages (Figure 7C). Indeed, several studies have demonstrated that cellular phagocytosis over time influences macrophage phenotype, causing a switch from a proinflammatory to a growth-promoting, reparative phenotype (44-46). Interestingly, while the total number of CD8 $8^{+}$tumor-infiltrating lymphocytes was reduced by prolonged combination therapy, the relative percentage of cytotoxic T cells was greatly increased (Supplemental Figure 7), possibly suggesting a boost in overall tumor-specific T cell frequency. 
In this manner, this combination therapy may allow for enhanced tumor antigen presentation at the earlier time points of treatment through increasing tumor phagocytosis and antigen uptake, while prolonged treatment limits general $\mathrm{T}$ cell infiltration after progression to a wound-healing TAM phenotype. Future experiments using trastuzumab plus anti-CD47 mAbs analyzing multiple treatment time points, reducing the length of treatment, or combining with other immune checkpoint blockades could potentially improve the infiltration of tumor-specific cytotoxic $\mathrm{T}$ lymphocytes.

Although this is an area in need of additional study, our results suggest that strategies to specifically enhance ADCP activity may be critical in overcoming resistance to HER2 mAb therapies by inhibiting tumor growth and potentially enhancing antigen presentation. While only a single clinical trial using a combination of therapeutic $\mathrm{mAb}$ (anti-CD20) and CD47 $\mathrm{mAb}$ has been reported, this study demonstrated an approximately 50\% response rate (11 of 22 patients) and an approximately $36 \%$ complete response rate ( 8 of 22 patients) in resistant/refractory non-Hodgkin lymphoma (41). These clinical findings, in conjunction with our recent preclinical studies, strongly suggest that the combination therapy approach of trastuzumab with CD47/SIPR $\alpha$ checkpoint blockade could potentially show more benefits and insights into trastuzumab therapy in HER2 ${ }^{+}$BC patients. However, the transcriptional switch seen in macrophages after prolonged ADCP also requires attention in future studies that utilize CD47 blockade in combination with targeted mAbs.

In sum, our study suggests that the dominant therapeutic MOA for trastuzumab is through its elicitation of TAM-mediated ADCP, which can be enhanced by strategies to specifically augment ADCP. This has potential implications for the use of trastuzumab in $\mathrm{HER} 2^{+}$cancers, as well as the utilization of other targeted therapies (such as EGFR, CD20, etc.), where efforts to enhance and control ADCP have not been prioritized.

\section{Methods}

Cell lines and genetic modification strategies. Mouse mammary gland cell lines MM3MG, NMUMG, and EPH4 were obtained from ATCC and cultured according to ATCC protocols. The cDNA of a naturally occurring splice variant of human HER2 (HER2A16) or wild-type HER2 was transduced into MM3MG and NMUMG cells using lentiviral transduction. The human HER2+ breast cancer cell line KPL4 was a gift from Junichi Kurebayashi (University of Kawasaki Medical School, Kurashiki, Japan) (64) and SKBR3 cells were purchased from ATCC and cultured according to ATCC's instructions. The Jurkat-NFAT-LUC line was obtained from Invivogen (jktl-nfat). CRISPR/Cas9 was used to knock out mouse Cd47 in MM3MGHER $2 \triangle 16$ cells or human CD47 in KPL4 cells. Gene targeting of mouse Cd47, human CD47, and control gene GFP by CRISPR/Cas9 was accomplished through the use of pLentiCRISPRv2 (Addgene plasmid 52961) using published protocols (65). Genes were targeted using the guide sequences CCCTTGCATCGTCCGTAATG and GGATAAGCGCGATGCCATGG for mouse Cd47, ATCGAGCTAAAATATCGTGT and CTACTGAAGTATACGTAAAG for human CD47, and GGGCGAGGAGCTGTTCACCG for the GFP control. Successful targeting of CD47 was determined by flow cytometry screening after single-cell clonal selection. The overexpression vector for mouse $C d 47$ was generated by synthesizing the $C d 47$ gene and subcloning it into pENTR1a (using NEB Gibson Isothermal Assembly Mix) and then using L/R clonase to generate expression lentiviruses (pLenti-CMV-Puro); cells were selected using puromycin.

Mice. Female BALB/c (Jackson Labs, stock 000651), SCID-beige (C.B-Igh-1b/GbmsTac-Prkdc ${ }^{\text {sid }}$-Lyst ${ }^{\text {bg }}$ N7; Taconic Biosciences, CBSCBG), Fcer1g ${ }^{-/}$[C.129P2(B6)-Fcerlg ${ }^{\text {tmlRav }}$ N12; Taconic Biosciences, 584] mice between the ages of 6 and 10 weeks were used for all experiments. The HER2 16 transgenic model was generated by crossing the MMTV-rtTA strain with the TetO-HER2d16-IRES-EGFP strain (20). Six-weekold mice were put on a doxycycline diet and enrolled for experiments when they developed palpable breast tumors (usually 4-6 weeks after the doxycycline diet).

Therapeutic antibodies and other experimental reagents. Clinical-grade trastuzumab (human IgG1) was obtained from Duke Medical Center. 4D5, the murine version of trastuzumab (with the IgG2A and IgG1 mouse isotypes) was produced by GenScript by special request. CD47 blockade antibody MIAP410 (BE0283) and control mouse IgG2A (BE0085) were purchased from BioXCell. Neutrophil-depleting antiLY6G (IA8, BP0075-1) and macrophage-depleting anti-CSF1R (AS598, BE0213) antibodies were purchased from BioXCell. Clodronate liposomes were purchased from www.ClodronateLiposomes.org.

Orthotopically implanted $H E R 2^{+} B C$ mouse models and therapeutic antibody treatments. MM3MG cells expressing human HER2 $\triangle 16$ were implanted into the mammary fat pads (MFPs) $\left(1 \times 10^{6}\right.$ cells) of BALB/c mice. For the human xenograft model, KPL-4 cells $\left(1 \times 10^{6}\right.$ cells $)$ were implanted into MFPs of SCID-beige BALB/c mice. Tumor growth was measured using caliper-based tumor volume (length $\times$ width $\times$ depth) over time. 
For therapeutic treatments, trastuzumab or 4D5 was administered weekly (200 $\mu$ g per mouse intraperitoneally) around 4 or 5 days after tumor implantation. CD47 blockade (MIAP410) was administered weekly when indicated (300 $\mu \mathrm{g}$ per mouse intraperitoneally) around 4 or 5 weeks after tumor implantation. For macrophage depletion, anti-CSF1R antibody was administered triweekly (300 $\mu \mathrm{g}$ per mouse intraperitoneally), starting at 2 weeks before tumor implantation and with treatment maintained over the course of the experiment. Clodronate liposomes were administered biweekly (100 $\mu \mathrm{L}$ per mouse, intraperitoneally). For neutrophil depletion, anti-LY6G antibody was administered biweekly (300 $\mu$ g per mouse intraperitoneally) for the first 2 weeks after tumor implantation.

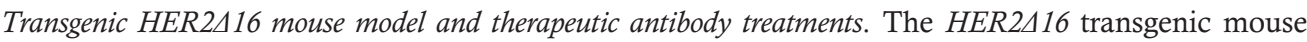
model was generated by crossing 2 strains of mice, TetO-HER2 $16-I R E S-E G F P$ and MMTV-rtTA. This system was described previously (20), but utilizes a TET-ON system (with MTV-rtTA) to drive expression of HER $2 \triangle 16$ to generate HER2 ${ }^{+}$BC. For experiments, 1-month-old mice were put on a doxycycline diet (200 $\mathrm{mg} / \mathrm{kg}$, Bio-Serv) to induce spontaneous HER2-driven BC. Individual animals were randomly enrolled into a specific treatment group as soon as palpable breast tumors were detected $\left(\sim 200 \mathrm{~mm}^{3}\right)$ in any of the 8 mammary fat pads. Control and 4D5-IgG2A antibodies were administered at $200 \mu \mathrm{g}$ weekly, whereas MIAP410 was administered at $300 \mu \mathrm{g} /$ week intraperitoneally. Animals were euthanized once their total tumor volume reached greater than $2000 \mathrm{~mm}^{3}$.

Flow cytometry analysis of tumor-infiltrating immune cells. When tumor growth reached humane end point size $\left(>1000 \mathrm{~mm}^{3}\right)$, whole tumors from mice were harvested and cut into small pieces $(<1 \mathrm{~mm})$, and incubated for 1 hour in digestion buffer (DMEM $+100 \mu \mathrm{g} / \mathrm{mL}$ collagenase $+0.2 \mathrm{U} / \mathrm{mL}$ DNAse +1 $\mu \mathrm{g} / \mathrm{mL}$ hyaluronidase). Single-cell suspensions were spun down through a $70-\mu \mathrm{m}$ filter and washed with medium. Approximately 5 million cells were used for staining and flow cytometry analysis. The following panel of immune cell markers (Biolegend) was used: CD45 BV650 (catalog 103140), CD11b PE-Cy7 (catalog 101216), LY6G APC (catalog 127614), LY6C BV410 (catalog 128032), F4/80 PerCP-CY5.5 (catalog 123128), CD8B APC-CY7 (catalog 126620), CD4 PE-TR (catalog 1005666), CD49b FITC (catalog 108906), and viability dye (Aqua or Red; Thermo Fisher Scientific). TAMs were identified by

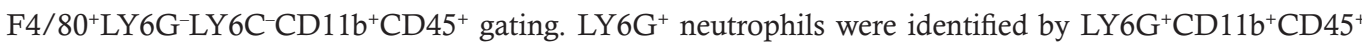
gating, whereas $\mathrm{LY}_{6 \mathrm{C}^{+}}$monocytes were gated on $\mathrm{LY} 6 \mathrm{C}^{+} \mathrm{CD} 11 \mathrm{~b}^{+} \mathrm{CD} 45^{+}$cells.

In vivo ADCP assay. MM3MG-HER2 $\triangle 16$ cells were labeled with Vybrant DiD labeling solution (Thermo Fisher Scientific, V22887) according to the manufacturer's protocol, and labeled cells were implanted $\left(1 \times 10^{6}\right)$ into MFPs of BALB/c mice. Once tumors reached approximately $1000 \mathrm{~mm}^{3}$, mice were treated with control antibody (200 $\mu \mathrm{g}), 4 \mathrm{D} 5(200 \mu \mathrm{g})$, or 4D5 in combination with MIAP410 (300 $\mu \mathrm{g})$ each day for 2 consecutive days. TAMs were analyzed by FACS $\left(\mathrm{CD} 11 \mathrm{~b}^{+} \mathrm{F} 4 / 80^{+} \mathrm{LY}_{6 \mathrm{G}}^{-} \mathrm{LY}^{-} \mathrm{C}^{-}\right)$and the percentage of TAMs that had taken up DiD-labeled tumor cells was quantified for in vivo ADCP analysis.

In vitro $A D C P$ and $A D C C$ assays. $\mathrm{ADCP}$ and $\mathrm{ADCC}$ by macrophages: $\mathrm{BMDMs}$ were generated from mouse tibia, differentiated for 10 days with $50 \mathrm{ng} / \mathrm{mL}$ mouse MCSF (Peprotech, 315-02). Briefly, 50 million bone marrow cells were plated in $10-\mathrm{cm}^{2}$ tissue culture dishes with MCSF on day 0 . Unattached cells in supernatant were removed and fresh media plus MCSF were added on days 3, 6, and 9. BMDMs were used for ADCP/ADCC assays on day 10. MM3MG-HER2 $\triangle 16$ tumor cells were labeled with Brilliant Violet 450 Dye (BD, 562158) according to the manufacturer's protocol, and incubated with control or anti-HER2 antibodies $(10 \mu \mathrm{g} / \mathrm{mL})$ in 96 -well plates $\left(100,000\right.$ cells/well) for 30 minutes at $37^{\circ} \mathrm{C}$. BMDMs were then added for coculture at a 3:1 ratio of tumor cells/BMDMs. After 2 hours of coculture, phagocytosis of BV450-labeled tumor cells by BMDMs was analyzed by FACS with CD45-APC staining and LIVE/DEAD (Red) staining (Thermo Fisher Scientific). When indicated, ADCP inhibitor latrunculin A (120 nM; Thermo Fisher Scientific, L12370) and ADCC inhibitor concanamycin A (1 $\mu \mathrm{M}$; Sigma-Aldrich, C9705) were added as assay controls. For human macrophage ADCP assays, human monocyte-derived macrophages (hMDMs) were generated from 3 donors' PBMCs. hMDMs were generated with $50 \mathrm{ng} / \mathrm{mL}$ human MCSF (Peprotech, 300-25) and $50 \mathrm{ng} / \mathrm{mL}$ human GM-CSF (Peprotech, 300-03). KPL-4 cells were used as human HER2 ${ }^{+}$ tumor targets and labeled and cocultured similarly to those used for mouse ADCP assays.

FCGR binding/activation assay. Jurkat cells with NFAT-luciferase reporter were transduced with lentiviral constructs to express mouse $F_{c g r} 1, F_{c g r} 2 b, F c g r 3$, or Fcgr4, and selected with puromycin and validated in Supplemental Figure 4, D-F. For the assay, MM3MG BC lines expressing HER2 were first plated and treated with trastuzumab or 4D5 antibodies or control IgG for 1 hour. Jurkat-FCGR-NFAT-LUC effector cells were added and cocultured for 4 hours. FCGR signaling activation was assessed by luciferase activity quantification. 
Multiplex cytokine and chemokine assay. BMDMs were cocultured with MM3MG-HER2 16 cells for 24 hours, and supernatants were harvested for analysis of cytokines/chemokines levels. The 26-Plex Mouse ProcartaPlex Panel 1 kit (Thermo Fisher Scientific) was used and analyzed using the Luminex MAGPIX system.

METABRIC analysis of CD47 expression in BC patients. Preprocessing METABRIC data: Previously normalized gene expression and clinical data were obtained from the European Genome-Phenome Archive (EGA) under the accession ID EGAS00000000098 after appropriate permission from the authors (47). The discovery data set was composed of 997 primary breast tumors and a second validation set was composed of 995 primary breast tumors. The expression data were arrayed on an Illumina HT12 Bead Chip composed of 48,803 transcripts. Multiple exon-level probe sets from a transcript cluster grouping were aggregated to a single-gene-level probe set using maximum values across all the probes for a given gene. The resulting gene expression matrix consists of 28,503 genes.

In order to assess the prognostic significance of $C D 47$ in METABRIC data we generated Kaplan-Meier survival curves for patients stratified by the average expression of CD47 (into low and high groups) using the R package survminer (version 0.4.3). Distributions of Kaplan-Meier survival curves for progression-free and overall survival were compared using the log-rank test, and a log-rank test $P$ value of 0.05 or less was considered to be statistically significant.

Single-cell RNA sequencing analysis. Fastq files from 10× library sequencing were processed using the CellRanger pipeline available from $10 \times$ Genomics. As part of the processing, the assembled sequencing reads were mapped to the mm10 mouse genome. In order to obtain the transcript counts of human ERBB2 (HER2) the sequencing reads were separately aligned to the current version of the human genome, GRCh38.

The gene expression files consisting of raw counts at the gene level for each cell was analyzed using version 2.3.4 of the Seurat package. The human ERBB2 counts were combined with the mm10-based counts into one expression matrix for each sample. Briefly, the data analysis steps using Seurat consisted of combining the gene counts for all the cells in the different conditions into 1 matrix, filtering low quality cells, normalizing, and adjusting for cell cycle and batch effects. Unsupervised clustering was done to separate the cell types and markers for the cell types that were identified using differential gene expression. These markers were then used for identifying the cell subpopulations within the tumor microenvironment, namely the immune cells, tumor cells, and fibroblasts. The normalized gene counts were used to generate tSNE maps for visualization of the cell types and heatmaps for the cell type-specific gene expression. Expression of predefined gene sets representing pathways of interest were obtained from previous publications and are summarized in Supplemental Table 2. The data discussed in this publication have been deposited in NCBI's Gene Expression Omnibus and are accessible through GEO Series accession number GSE139492 (https://www.ncbi.nlm.nih.gov/geo/query/acc.cgi?acc=GSE139492).

Statistics. All statistical analyses of tumor growth comparisons and tumor immune infiltrates were performed with GraphPad Prism (v8) using 2-way ANOVA or 1-way ANOVA with Tukey's multiple-comparisons test. Unless otherwise indicated in the figure, test results are shown for treatment versus the control group. Group sizes for animal tumor growth experiments were determined based on preliminary data sets. All subjects in animal experiments were randomized into a treatment or control group. For in vitro experiments, i.e., ADCP, ADCC, and CDC assays, ELISPOT assays, FCGR signaling assays, and cytokine assays, all data were statistically analyzed by 1-way ANOVA test with Tukey's multiple-comparisons test, and performed with at least 4 biological replicates per experiment and repeated at least 2 times. RT-qPCR data were analyzed by 2 -sided Student's $t$ test for each target gene. The $95 \%$ confidence interval was considered for statistics and $P$ less than 0.05 was considered significant.

Study approval. All animals were maintained and bred in accordance with the Duke Institutional Animal Care and Use Committee-approved protocol (A198-18-08), and supervised by Division of Laboratory Animal Resources (DLAR).

\section{Author contributions}

LCT, HKL, and ZCH conceived the project and wrote the manuscript. LCT and ZCH designed the experiments and analyzed and interpreted the data. LCT performed the animal experiments, with assistance from EJC, BJW, XY, CXL, and CAR. TW provided technical support and suggestions for flow cytometry. ZCH generated various cell lines and CRISPR knockouts with assistance from JW and GL. LCT performed the in vitro cell culture assays, designed single-cell RNA sequencing experiment, and generated 10× Genomics 
cDNA libraries. PA performed the clustering and statistical analysis and illustration of single-cell RNA sequencing results. CA conducted the stratification and statistical analysis of METABRIC datasets. EJC, TNT, and CWS provided intellectual input and discussions. LAC provided the MMTV-rtTA mouse strain and WJM provided the TetO-HER2d16-IRES-EGFP mouse strain.

\section{Acknowledgments}

This research was supported by grants from the NIH (5K12CA100639-09 to ZCH, 1R01CA238217-01A1 to ZCH) and (T32CA009111 to LCT), Department of Defense (DOD) (BC113107 to HKL), Merck MISP Program (LKR160831 to ZCH), and Susan G. Komen (CCR14299200 to ZCH). We would also like to acknowledge all of the members of the Applied Therapeutics Center for their generous technical assistance and insights into this project.

Address correspondence to: Zachary C. Hartman, Department of Surgery and Pathology, Division of Surgical Sciences, Center for Applied Therapeutics, Duke University Medical Center, MSRBI Room 414 Box 2606, 203 Research Drive, Durham, North Carolina 27710, USA. Phone: 919.613.9110; Email: zachary.hartman@duke.edu.

1. Slamon DJ, Clark GM, Wong SG, Levin WJ, Ullrich A, McGuire WL. Human breast cancer: correlation of relapse and survival with amplification of the HER-2/neu oncogene. Science. 1987;235(4785):177-182.

2. Di Fiore PP, Pierce JH, Kraus MH, Segatto O, King CR, Aaronson SA. erbB-2 is a potent oncogene when overexpressed in NIH/3T3 cells. Science. 1987;237(4811):178-182.

3. Hudis CA. Trastuzumab--mechanism of action and use in clinical practice. NEngl J Med. 2007;357(1):39-51.

4. Untch M, et al. Neoadjuvant treatment with trastuzumab in HER2-positive breast cancer: results from the GeparQuattro study. J Clin Oncol. 2010;28(12):2024-2031.

5. Gianni L, et al. 5-year analysis of neoadjuvant pertuzumab and trastuzumab in patients with locally advanced, inflammatory, or early-stage HER2-positive breast cancer (NeoSphere): a multicentre, open-label, phase 2 randomised trial. Lancet Oncol. 2016;17(6):791-800.

6. de Azambuja E, et al. Lapatinib with trastuzumab for HER2-positive early breast cancer (NeoALTTO): survival outcomes of a randomised, open-label, multicentre, phase 3 trial and their association with pathological complete response. Lancet Oncol. 2014;15(10):1137-1146.

7. Bianchini G, Gianni L. The immune system and response to HER2-targeted treatment in breast cancer. Lancet Oncol. 2014;15(2):e58-e68.

8. Park S, et al. The therapeutic effect of anti-HER2/neu antibody depends on both innate and adaptive immunity. Cancer Cell. 2010;18(2):160-170.

9. Stagg J, et al. Anti-ErbB-2 mAb therapy requires type I and II interferons and synergizes with anti-PD-1 or anti-CD137 mAb therapy. Proc Natl Acad Sci USA. 2011;108(17):7142-7147.

10. Clynes RA, Towers TL, Presta LG, Ravetch JV. Inhibitory Fc receptors modulate in vivo cytotoxicity against tumor targets. Nat Med. 2000;6(4):443-446.

11. Musolino A, et al. Immunoglobulin G fragment C receptor polymorphisms and efficacy of preoperative chemotherapy plus trastuzumab and lapatinib in HER2-positive breast cancer. Pharmacogenomics J. 2016;16(5):472-477.

12. Muntasell A, et al. Interplay between natural killer cells and Anti-HER2 antibodies: perspectives for breast cancer immunotherapy. Front Immunol. 2017;8:1544.

13. Muntasell A, et al. NK cell infiltrates and HLA class I expression in primary HER2 ${ }^{+}$breast cancer predict and uncouple pathological response and disease-free survival. Clin Cancer Res. 2019;25(5):1535-1545.

14. Albanesi M, et al. Neutrophils mediate antibody-induced antitumor effects in mice. Blood. 2013;122(18):3160-3164.

15. Mortenson ED, Park S, Jiang Z, Wang S, Fu YX. Effective anti-neu-initiated antitumor responses require the complex role of CD4+ T cells. Clin Cancer Res. 2013;19(6):1476-1486.

16. Shi Y, et al. Trastuzumab triggers phagocytic killing of high HER2 cancer cells in vitro and in vivo by interaction with Fc $\gamma$ receptors on macrophages. J Immunol. 2015;194(9):4379-4386.

17. Su S, et al. Immune checkpoint inhibition overcomes ADCP-induced immunosuppression by macrophages. Cell. 2018;175(2):442-457.e23.

18. Bruhns P, Jönsson F. Mouse and human FcR effector functions. Immunol Rev. 2015;268(1):25-51.

19. Nimmerjahn F, Ravetch JV. Divergent immunoglobulin g subclass activity through selective Fc receptor binding. Science. 2005;310(5753):1510-1512.

20. Turpin J, et al. The ErbB2 $\Delta \mathrm{Ex} 16$ splice variant is a major oncogenic driver in breast cancer that promotes a pro-metastatic tumor microenvironment. Oncogene. 2016;35(47):6053-6064.

21. Crosby EJ, et al. Complimentary mechanisms of dual checkpoint blockade expand unique T-cell repertoires and activate adaptive anti-tumor immunity in triple-negative breast tumors. Oncoimmunology. 2018;7(5):e1421891.

22. Jaiswal S, Chao MP, Majeti R, Weissman IL. Macrophages as mediators of tumor immunosurveillance. Trends Immunol. 2010;31(6):212-219

23. Mantovani A, Longo DL. Macrophage checkpoint blockade in cancer - back to the future. NEngl J Med. 2018;379(18):1777-1779. 24. Huang Y, Ma Y, Gao P, Yao Z. Targeting CD47: the achievements and concerns of current studies on cancer immunotherapy. 
J Thorac Dis. 2017;9(2):E168-E174.

25. Zhang H, et al. HIF-1 regulates CD47 expression in breast cancer cells to promote evasion of phagocytosis and maintenance of cancer stem cells. Proc Natl Acad Sci USA. 2015;112(45):E6215-E6223.

26. Yan M, Schwaederle M, Arguello D, Millis SZ, Gatalica Z, Kurzrock R. HER2 expression status in diverse cancers: review of results from 37,992 patients. Cancer Metastasis Rev. 2015;34(1):157-164.

27. Carter P, et al. Humanization of an anti-p185HER2 antibody for human cancer therapy. Proc Natl Acad Sci USA. 1992;89(10):4285-4289.

28. Stewart R, Hammond SA, Oberst M, Wilkinson RW. The role of Fc gamma receptors in the activity of immunomodulatory antibodies for cancer. J Immunother Cancer. 2014;2(1):1-10.

29. Moasser MM. Two dimensions in targeting HER2. J Clin Oncol. 2014;32(19):2074-2077.

30. Moasser MM, Krop IE. The evolving landscape of HER2 targeting in breast cancer. JAMA Oncol. 2015;1(8):1154-1161.

31. Wang Y, et al. CD55 and CD59 expression protects HER2-overexpressing breast cancer cells from trastuzumab-induced complement-dependent cytotoxicity. Oncol Lett. 2017;14(3):2961-2969.

32. Gordon SR, et al. PD-1 expression by tumour-associated macrophages inhibits phagocytosis and tumour immunity. Nature. 2017;545(7655):495-499.

33. Willingham SB, et al. The CD47-signal regulatory protein alpha (SIRPa) interaction is a therapeutic target for human solid tumors. Proc Natl Acad Sci USA. 2012;109(17):6662-6667.

34. Oliveira CA, Kashman Y, Mantovani B. Effects of latrunculin A on immunological phagocytosis and macrophage spreading-associated changes in the F-actin/G-actin content of the cells. Chem Biol Interact. 1996;100(2):141-153.

35. Toda H, Araki K, Moritomo T, Nakanishi T. Perforin-dependent cytotoxic mechanism in killing by CD8 positive T cells in ginbuna crucian carp, Carassius auratus langsdorfii. Dev Comp Immunol. 2011;35(1):88-93.

36. Guilliams M, Bruhns P, Saeys Y, Hammad H, Lambrecht BN. The function of Fcy receptors in dendritic cells and macrophages Nat Rev Immunol. 2014;14(2):94-108.

37. Bournazos S, Wang TT, Ravetch JV. The role and function of Fcy receptors on myeloid cells. Microbiol Spectr. 2016;4(6).

38. Nimmerjahn F, et al. Fc $\gamma$ RIV deletion reveals its central role for IgG2a and IgG2b activity in vivo. Proc Natl Acad Sci USA 2010;107(45):19396-19401.

39. Parekh BS, et al. Development and validation of an antibody-dependent cell-mediated cytotoxicity-reporter gene assay. MAbs. 2012;4(3):310-318.

40. Dekkers G, et al. Affinity of human IgG subclasses to mouse Fc gamma receptors. MAbs. 2017;9(5):767-773

41. Advani R, et al. CD47 blockade by Hu5F9-G4 and rituximab in non-Hodgkin's lymphoma. N Engl J Med. 2018;379(18):1711-1721.

42. Lawson MA, et al. Osteoclasts control reactivation of dormant myeloma cells by remodelling the endosteal niche. Nat Commun. $2015 ; 6: 8983$.

43. Turpin J, et al. The ErbB2 2 Ex16 splice variant is a major oncogenic driver in breast cancer that promotes a pro-metastatic tumor microenvironment. Oncogene. 2016;35(47):6053-6064.

44. Fadok VA, Bratton DL, Konowal A, Freed PW, Westcott JY, Henson PM. Macrophages that have ingested apoptotic cells in vitro inhibit proinflammatory cytokine production through autocrine/paracrine mechanisms involving TGF-beta, PGE2, and PAF. J Clin Invest. 1998;101(4):890-898.

45. Lech M, Anders HJ. Macrophages and fibrosis: How resident and infiltrating mononuclear phagocytes orchestrate all phases of tissue injury and repair. Biochim Biophys Acta. 2013;1832(7):989-997.

46. Minutti CM, Knipper JA, Allen JE, Zaiss DM. Tissue-specific contribution of macrophages to wound healing. Semin Cell Dev Biol. 2017;61:3-11.

47. Curtis C, et al. The genomic and transcriptomic architecture of 2,000 breast tumours reveals novel subgroups. Nature. 2012;486(7403):346-352.

48. Kwong LS, Brown MH, Barclay AN, Hatherley D. Signal-regulatory protein $\alpha$ from the NOD mouse binds human CD47 with an exceptionally high affinity-- implications for engraftment of human cells. Immunology. 2014;143(1):61-67.

49. Hurvitz SA, et al. Analysis of Fcy receptor IIIa and IIa polymorphisms: lack of correlation with outcome in trastuzumab-treated breast cancer patients. Clin Cancer Res. 2012;18(12):3478-3486.

50. Barok M, et al. Trastuzumab causes antibody-dependent cellular cytotoxicity-mediated growth inhibition of submacroscopic JIMT-1 breast cancer xenografts despite intrinsic drug resistance. Mol Cancer Ther. 2007;6(7):2065-2072.

51. Ritter CA, et al. Human breast cancer cells selected for resistance to trastuzumab in vivo overexpress epidermal growth factor receptor and ErbB ligands and remain dependent on the ErbB receptor network. Clin Cancer Res. 2007;13(16):4909-4919.

52. Yakes FM, Chinratanalab W, Ritter CA, King W, Seelig S, Arteaga CL. Herceptin-induced inhibition of phosphatidylinositol-3 kinase and Akt Is required for antibody-mediated effects on p27, cyclin D1, and antitumor action. Cancer Res. 2002;62(14):4132-4141.

53. Grugan KD, et al. Tumor-associated macrophages promote invasion while retaining Fc-dependent anti-tumor function. J Immunol. 2012;189(11):5457-5466.

54. Gül N, van Egmond M. Antibody-dependent phagocytosis of tumor cells by macrophages: a potent effector mechanism of monoclonal antibody therapy of cancer. Cancer Res. 2015;75(23):5008-5013.

55. Brown JM, Recht L, Strober S. The promise of targeting macrophages in cancer therapy. Clin Cancer Res. 2017;23(13):3241-3250.

56. Ruffell B, Affara NI, Coussens LM. Differential macrophage programming in the tumor microenvironment. Trends Immunol. 2012;33(3):119-126

57. Noy R, Pollard JW. Tumor-associated macrophages: from mechanisms to therapy. Immunity. 2014;41(1):49-61.

58. Zhang QW, et al. Prognostic significance of tumor-associated macrophages in solid tumor: a meta-analysis of the literature. PLoS ONE. 2012;7(12):e50946

59. Mantovani A, Marchesi F, Malesci A, Laghi L, Allavena P. Tumour-associated macrophages as treatment targets in oncology. Nat Rev Clin Oncol. 2017;14(7):399-416

60. Taylor C, et al. Augmented HER-2 specific immunity during treatment with trastuzumab and chemotherapy. Clin Cancer Res. 2007;13(17):5133-5143 
61. Abès R, Gélizé E, Fridman WH, Teillaud JL. Long-lasting antitumor protection by anti-CD20 antibody through cellular immune response. Blood. 2010;116(6):926-934.

62. Pfirschke C, et al. Immunogenic chemotherapy sensitizes tumors to checkpoint blockade therapy. Immunity. 2016;44(2):343-354.

63. Chao MP, et al. Anti-CD47 antibody synergizes with rituximab to promote phagocytosis and eradicate non-Hodgkin lymphoma. Cell. 2010;142(5):699-713.

64. Kurebayashi J, et al. Isolation and characterization of a new human breast cancer cell line, KPL-4, expressing the Erb B family receptors and interleukin-6. Br J Cancer. 1999;79(5-6):707-717.

65. Shalem O, et al. Genome-scale CRISPR-Cas9 knockout screening in human cells. Science. 2014;343(6166):84-87. 